\title{
Tidal evolution of eccentric orbits in massive binary systems
}

\section{Coupled resonance locking for two rotating main sequence stars}

\author{
M. G. Witte and G. J. Savonije
}

\begin{abstract}
Astronomical Institute "Anton Pannekoek", University of Amsterdam, Kruislaan 403, 1098 SJ Amsterdam, The Netherlands

Received 8 September 2000 / Accepted 13 November 2000

Abstract. We extend our study of the tidal evolution of elliptic binary systems to the case of a system consisting of two $10 M_{\odot}$ uniformly rotating main sequence stars. Previous work showed that in a system consisting of a $1.4 M_{\odot}$ compact object in orbit about a $10 M_{\odot}$ main sequence star stellar oscillation modes were prone to be excited nearly resonantly for prolonged periods of time. This resonance locking was shown to constitute an effective mechanism for orbital decay in moderately eccentric orbits. In this work we investigate in what ways the locking mechanism is altered if also the companion is tidally perturbed, also inducing orbital changes and potentially also being locked in resonance. We show that simultaneous locking can intensify the tidal excitations, and that resonance locking is a common phenomenon during the tidal evolution of eccentric double main sequence binary systems which speeds up the secular evolution significantly.
\end{abstract}

Key words. stars: rotation - stars: oscillations - stars: binaries: close - hydrodynamics

\section{Introduction}

A study on the eccentricity of orbits in a sample of $\approx 200$ early type binaries by Giuricin et al. (1984) led them to the conclusion that the observed distribution of nearly circular orbits was "substantially compatible" with the asymptotic theory of radiatively damped dynamical tides proposed by Zahn (1977). There is, however, a significant fraction of almost circularized early type binaries with periods up to about 10 days which cannot be explained by Zahn's theory and which are unlikely to be primordial $e \simeq 0$ binaries. However, at higher eccentricities binary stars are subject to higher (harmonic) forcing frequencies for which Zahn's asymptotic low frequency approximation is inadequate and predicts far too long tidal evolution timescales (Savonije \& Papaloizou 1983).

We consider the tidal evolution of binary stars in eccentric orbit by decomposing the perturbing tidal potential into its harmonic components, and by calculating the torque on the two stars as the linear superposition of the torques induced by these forcing harmonics (see Witte \& Savonije 1999b, Paper II). The non-adiabatic stellar response to each harmonic is calculated numerically (Witte \& Savonije 1999a, Paper I) using a 2D implicit hydrodynamic code developed by Savonije \& Papaloizou (1997), which takes Coriolis forces due to the stellar rotation fully into account. Nonradial ( $g$ - and $r-$ ) modes of the stars

Send offprint requests to: M. G. Witte,

e-mail: marnix@astro.uva.nl may be resonantly excited, strongly enhancing the tidal exchange of energy and angular momentum between the two stars. It is often argued that such (nearly) resonant tidal interaction can be neglected because once at resonance the strongly enhanced tidal dissipation will rapidly change the stellar spin and the orbit, so that the narrow resonance condition is almost immediately lost and only limited exchange of energy and angular momentum and energy can take place. However, in Paper II we showed that - when stellar rotation is taken into account - one of the orbital harmonics in eccentric binaries may become locked on a resonance. During such locking the nearly resonant interaction can be sustained for a prolonged period of time even though the orbit and stellar spin change significantly. Such phases of rapid orbital evolution will last until the orbital eccentricity has decayed so much that the power re-distribution in the orbital harmonics becomes too large or until some other orbital frequency approaches a resonance, tilting the balance and pushing the locked harmonic through or back out of resonance.

Besides providing efficient orbital evolution, resonance locking may be of interest to those trying to interpret observed oscillations of binary components (see Smith 1985a,b; Harmanec et al. 1997; Willems et al. 1997; De Cat et al. 2000), because during a period of resonance locking a certain harmonic of the orbital frequency will be almost equal to one of the eigenfrequencies in the stellar eigenspectrum. A preferred coincidence of orbital harmonic frequencies with stellar oscillation frequencies could 
be a great benefit in the asteroseismological research of binary components. Observation of enhanced tidal interaction yields the approximate eigenfrequency (but in the inertial frame, not in the stellar frame) of a damped oscillation mode which without tidal forcing would not self-excite and could therefore not be studied.

In Paper II we concentrated on the evolution of binaries consisting of a pointmass-like (neutron star) companion orbiting a heavy $\left(10 M_{\odot}\right)$ main sequence (MS) star. In this paper we describe the orbital evolution of a binary system consisting of two $10 M_{\odot}$ MS stars. In such case the angular momentum distribution in the system at a given binary period is different, because the larger companion mass implies that the orbital moment of inertia is larger. Now the second MS star also has a large moment of inertia and it also takes part in the tidal dissipation. Inclusion of a second spectrum of stellar oscillation modes adds to the complexity of the system and may influence the delicate process of resonance locking. We will now first summarise the formulae that describe orbital evolution and resonance locking in double MS binaries.

\section{Basic equations}

We consider a binary system consisting of two $M_{i}=$ $10 M_{\odot}$ MS stars in an eccentric orbit with eccentricity $e$ and orbital period $P_{\text {orb }}$. The energy and angular momentum (magnitude) of the eccentric orbit is given by

$E_{\mathrm{orb}}=-\frac{G M_{1} M_{2}}{2 a}$ and $H_{\mathrm{orb}}=\frac{M_{1} M_{2}}{M_{1}+M_{2}} a^{2} \omega \sqrt{1-e^{2}}$

where $a$ is the semi-major axis and $\omega=2 \pi / P_{\text {orb }}$ the mean angular velocity of the stars in their elliptic orbit. For simplicity we assume the stellar spin angular momentum vectors $\boldsymbol{H}_{1}$ and $\boldsymbol{H}_{2}$ to be aligned with the orbital angular momentum vector. As in Paper II, we will only consider secular tidal changes in the magnitude of the orbital energy and angular momentum, and ignore changes in the configuration of the orbit as given by the advance of periastron (apsidal motion). We briefly summarise what relations were established in Paper II, and indicate how the relations for resonance locking are altered when both stars contribute to the tidal dissipation.

\subsection{Equations of tidal evolution}

For each star its companion's tidal potential can be expressed as

$$
\begin{gathered}
\Phi_{\mathrm{T}}(r, \vartheta, \varphi, t)=-\sum_{l=2}^{\infty} \sum_{m=0}^{l} r^{l} P_{l}^{m}(\cos \vartheta) \\
\sum_{n=-\infty}^{\infty} c_{n}^{l m} \cos (n \omega t-m \varphi),
\end{gathered}
$$

where $(r, \vartheta, \varphi)$ are spherical polar coordinates with the origin at the centre of mass of one of the stars and whereby
$P_{l}^{m}(\cos \vartheta)$ are the associated Legendre functions. The coefficients

$c_{n}^{l m}=\left(\frac{G M_{\text {other }}}{a^{l+1}}\right) \epsilon_{m} \frac{(l-m) !}{(l+m) !} P_{l}^{m}\left(\cos \frac{\pi}{2}\right) h_{n}^{(l+1), m}$

are given in terms of the Hansen coefficients

$h_{n}^{(l+1), m}=\frac{1}{2 \pi} \int_{-\pi}^{\pi}\left(\frac{a}{r^{\prime}}\right)^{l+1} \mathrm{e}^{\mathrm{i}\left(m \varphi^{\prime}-k M\right)} \mathrm{d} M$,

where $M=\omega t$ is the mean anomaly and primed coordinates give the location of the companion in its orbit. In the following we only consider the dominant $l=2$ contribution.

The response to the forcing by each potential term in summation (1) is assumed to have reached a steady state oscillation, i.e. its (harmonic) time dependence is known. The steady state approximation requires that the tidal evolution timescale remains much longer than the damping time $\tau_{\mathrm{d}}$ of the excited modes, and with typically $\tau_{\mathrm{d}} \lesssim 10^{2}$ yr this poses in general no problem. The steady state response to the forcing, in particular the density perturbation $\rho_{i}^{\prime}=\rho_{i}^{\prime}(r, \vartheta) \mathrm{e}^{\mathrm{i}(n \omega t-m \varphi)}$, is evaluated numerically with the $2 \mathrm{D}$ oscillation code. For any given forcing frequency one can thus evaluate the tidal interaction between the two stars in the form of a torque integral in terms of the density perturbation over each star $(i=1,2)$ :

$$
\begin{gathered}
\mathcal{T}_{i n}^{l m}=-\pi c_{n}^{l m} \int_{0}^{R_{i}} \int_{0}^{\pi} \operatorname{Im}\left(\rho_{i}^{\prime}(r, \vartheta)\right) . \\
P_{l}^{m}(\cos \vartheta) r^{l+2} \sin \vartheta \mathrm{d} \vartheta \mathrm{d} r
\end{gathered}
$$

where Im stands for imaginary part. Note that the torque integral can be either positive (excitation of prograde oscillation modes for $\bar{\sigma}_{n}=n \omega-m \Omega_{i}>0$ ) or negative (retrograde modes excited by the lower orbital harmonics), where $\bar{\sigma}_{n}$ is the forcing frequency in the stellar frame. The torque integral is evaluated numerically over the tidal response on the $2 \mathrm{D}(r, \vartheta)$ grid. The work done per unit time by the partial tide $(n, l, m)$ on star $i$ and the associated rate of change of the stellar spin momentum follows respectively as

$\dot{E}_{\text {in }}^{l m}=n \omega \mathcal{T}_{i n}^{l m} \quad$ and $\quad \dot{H}_{i n}^{l m}=m \mathcal{T}_{i n}^{l m}$

where again $i=1,2$ for the two stars.

The induced changes of orbital energy and angular momentum are then calculated by merely adding all the harmonic contributions of both stars together:

$\dot{E}_{\text {orb }}=-\sum_{i} \sum_{m} \sum_{n} \dot{E}_{i n}^{l m}=-\sum_{i} \dot{E}_{i}$
$\dot{H}_{\text {orb }}=-\sum_{i} \sum_{m} \sum_{n} \dot{H}_{i n}^{l m}=-\sum_{i} \dot{H}_{i}$

with $\dot{E}_{i}=\sum_{m} \sum_{n} \dot{E}_{i n}^{l m}$ and $\dot{H}_{i}=\sum_{m} \sum_{n} \dot{H}_{i n}^{l m}$ the rates of change of rotational energy and (spin) angular momentum for each of the stars $i$. The rates of change of the 
orbital eccentricity and the semi-major axis follow as

$$
\begin{aligned}
\frac{\mathrm{d} e^{2}}{\mathrm{~d} t} & =\left(\frac{G M_{1} M_{2}}{2 a}\right)^{-1}\left[\left(1-e^{2}\right) \dot{E}_{\text {orb }}-\omega \sqrt{1-e^{2}} \dot{H}_{\text {orb }}\right] \\
\frac{1}{a} \frac{\mathrm{d} a}{\mathrm{~d} t} & =\left(\frac{G M_{1} M_{2}}{2 a}\right)^{-1} \dot{E}_{\text {orb }},
\end{aligned}
$$

while the spin rate of star $i$ changes as

$$
\frac{\mathrm{d} \Omega_{i}}{\mathrm{~d} t}=\frac{1}{I_{i}} \dot{H}_{i}-\frac{H_{i}}{I_{i}^{2}} \dot{I}_{i} .
$$

Here, $I_{i}$ is the moment of inertia of star $i$, and $\dot{I}_{i}$ the rate at which this moment of inertia increases as the star expands during core hydrogen burning.

\subsection{Evaluation of torque integrals}

The steady state oscillation amplitude forced by a harmonic term in the tidal potential at a given frequency strongly depends on the spectrum of stellar oscillation modes. The value of the torque integral $\mathcal{T}$ is the largest when the forcing frequency is close to a stellar eigenfrequency. Near an eigenfrequency the torque integral over the volume of star $i$ due to harmonic forcing frequency $\sigma_{n}=n \omega$ can be represented by the resonance curve of a harmonic oscillator:

$\mathcal{T}_{\text {in }}^{l m}=\mathcal{Q}_{n} \mathcal{T}_{0, i k}^{l m}$

with

$\mathcal{Q}_{n}=\frac{1}{\left(\frac{\bar{\sigma}_{n}^{2}-\bar{\sigma}_{0, i k}^{2}}{\bar{\sigma}_{n} \Delta \bar{\sigma}_{i k}}\right)^{2}+1}$.

Here, $\bar{\sigma}_{0, i k}(l, m)$ is the resonance frequency of the stellar oscillation mode with symmetry $(l, m)$ and $k$ radial nodes, $\Delta \bar{\sigma}_{i k}(l, m)$ the peak width (FWHM) of the corresponding resonance curve and $\mathcal{T}_{0, i k}^{l m}$ the value of the torque integral at full resonance. Tables of resonance frequencies $\bar{\sigma}_{0, i k}$, peak widths $\Delta \bar{\sigma}_{i k}$ and peak heights $\mathcal{T}_{0, i k}^{l m}$ for $l=2$ and $m=$ 0,2 for a $10 M_{\odot}$ MS star at different rotation rates and different stages of stellar evolution are given in Papers I and II. Interpolations within these tables are performed to calculate the stellar oscillation spectra $\mathcal{T}_{\text {in }}^{l m}$ at each time step during the orbital evolution.

Going from high to low pro- or retrograde forcing frequencies in the stellar frame the wavelength of the stellar g-mode response decreases, increasing the number of radial nodes in the oscillation cavity between the convective core and the stellar surface. The frequency spacing between adjacent eigenfrequencies of stellar g-modes decreases, while the low frequency gravity waves can penetrate deeper towards the stellar surface where radiative damping is strong. As a result of the severe damping in the surface layers the low frequency g-mode resonance peaks become wider and lower. Eventually the stellar resonances become smeared out to form a relatively featureless torque level which varies only slowly with frequency, on top of which the narrow bunch of torque peaks due to (quasi-toroidal) r-mode resonances are superposed for slightly negative frequencies. For the orbital calculations the heavily damped g-modes with more than twenty radial nodes are not represented individually, and the torque distribution between prograde $g_{20}$ and retrograde $g_{-20}$ is approximated by a constant torque level $\mathcal{T}_{\text {low }}$ (except near corotation).

An average level of $\mathcal{T}_{\text {low }}=310^{37}$ cgs represents the results of our numerical calculations for the $X_{\mathrm{c}}=0.4$ stellar model best. In Paper II we used a detailed fit to the numerical result obtained in Paper I (down to the lowest frequencies) but here we use this simple prescription for the low frequency forcing which gives similar results. Note that near corotation the torque appears still rather large and it is only in a narrow region $|\bar{\sigma}|<0.01 \Omega_{i}$ that the torque rapidly falls off to zero (see Papaloizou \& Savonije 1997, and Paper I) and changes sign at $\bar{\sigma}=0$. In Sect. 3.4 we discuss how the orbital evolution calculations depend on the value of $\mathcal{T}_{\text {low }}$.

\subsection{Resonance locking in double MS binaries}

By applying Eqs. (3)-(9) the rate of change of the mean orbital frequency due to the tidal dissipation induced by the nearly resonant forcing of a certain orbital harmonic $(n, l, m)$ can be expressed as

$\dot{\omega}=\frac{3 n}{I_{\text {orb }}} \mathcal{T}_{\text {in }}^{l m}$

where the orbital moment of inertia $I_{\text {orb }}=\mu a^{2}$ with $\mu=$ $\frac{M_{1} M_{2}}{M_{1}+M_{2}}$. Note that in the inertial frame both stars are forced with the same harmonic frequencies $n \omega$. Therefore, in a double MS star binary the rate at which the forcing frequency in the stellar frame (symbolised by a bar) $\bar{\sigma}_{n}=$ $n \omega-m \Omega_{i}$ of harmonic $n$ drifts relative to the resonance frequency $\bar{\sigma}_{0, i k}$ of a certain oscillation mode $k$ of star $i$ can be written as

$$
\begin{aligned}
\frac{\mathrm{d}}{\mathrm{d} t} & \left(\bar{\sigma}_{n}-\bar{\sigma}_{0, i k}\right)=n \sum_{i^{\prime}, m^{\prime}, j} \frac{3 j}{I_{\mathrm{orb}}} \mathcal{T}_{i^{\prime} j}^{l m^{\prime}} \\
& -\sum_{m^{\prime}, j} \frac{m}{I_{i}}\left(m+\frac{\partial \bar{\sigma}_{0, i k}}{\partial \Omega_{i}}\right) \mathcal{T}_{i j}^{l m^{\prime}} \\
& +\left(\frac{\Omega_{i}}{I_{i}}\left(m+\frac{\partial \bar{\sigma}_{0, i k}}{\partial \Omega_{i}}\right) \frac{\mathrm{d} I_{i}}{\mathrm{~d} X_{i}}-\frac{\partial \bar{\sigma}_{0, i k}}{\partial X_{i}}\right) \dot{X}_{i}
\end{aligned}
$$

Here, the first term on the right yields the shifting rate of the orbital harmonics caused by the tidal dissipation in both stars, therefore this summation is over $i^{\prime}=1,2$. The second term reflects shifting due to tidal spin-up or spin-down of star $i$, including the rotational effect on the oscillation frequency spectrum of star $i$. The third term sums the shifting rate due to intrinsic spin-down of the star as a result of its increasing moment of inertia, and due to intrinsic changes in the stellar eigen frequency spectrum which occur as the star restructures during core hydrogen burning. This last term contains no torque, but is merely proportional to the rate $\dot{X}_{i}$ of hydrogen depletion in the 
core of star $i$. Following the notations adopted in Paper II we introduce

$\zeta_{\text {inj }} \equiv\left[\frac{3 n j}{I_{\text {orb }}}-\frac{m}{I_{i}}\left(m+\frac{\partial \bar{\sigma}_{0, i k}}{\partial \Omega_{i}}\right)\right]$

for the dynamical factors which appear in Eq. (13) and

$\epsilon_{i} \equiv\left[\frac{\Omega_{i}}{I_{i}}\left(m+\frac{\partial \bar{\sigma}_{0, i k}}{\partial \Omega_{i}}\right) \frac{\mathrm{d} I_{i}}{\mathrm{~d} X_{i}}-\frac{\partial \bar{\sigma}_{0, i k}}{\partial X_{i}}\right]$

for the stellar evolution factors. Note that for a harmonic of sufficiently large $j$ the sign of $\zeta_{i n j}$ becomes positive but that for the strongest harmonics $\zeta_{i n j}$ is negative, so that for some harmonic $\left|\zeta_{i n j}\right|$ can be small. To facilitate future references to the different terms which are present in Eq. (13), we will further introduce the following shorthand notations:

$\partial_{t, n}\left(\bar{\sigma}_{n}-\bar{\sigma}_{0, i k}\right) \equiv \zeta_{i n n} \mathcal{T}_{i n}^{l m}$

for the relative shift rate induced by the nearly resonant harmonic $n$ itself by the change of the orbital period and the spin-up/down of star $i$ it induces;

$\partial_{t, \not \chi}\left(\bar{\sigma}_{n}-\bar{\sigma}_{0, i k}\right) \equiv \sum_{m^{\prime}, j} \zeta_{i n j} \mathcal{T}_{i j}^{l m^{\prime}}-\zeta_{i n n} \mathcal{T}_{i n}^{l m}$

for the rate of shifting due to all tidally excited modes in star $i$, except the aforementioned (nearly resonant) excitation by harmonic $n$;

$\partial_{t, \dot{x}}\left(\bar{\sigma}_{n}-\bar{\sigma}_{0, i k}\right) \equiv \sum_{m^{\prime}, j} \frac{3 n j}{I_{\text {orb }}} \mathcal{T}_{i_{2} j}^{l m^{\prime}}$

for the rate of shifting of harmonic frequency $n$ relative to the eigenfrequency of mode $k$ in star $i$ due to changes in the orbital period caused by dissipation in the companion star $i \pm 1$;

$\partial_{t, \dot{X}}\left(\bar{\sigma}_{n}-\bar{\sigma}_{0, i k}\right) \equiv \epsilon_{i} \dot{X}_{i}$

for the rate of shifting due to stellar evolution, i.e. due to the stellar restructuring in response to hydrogen burning in the interior of star $i$. The total rate of frequency shifting relative to the resonance with harmonic $n$ (Eq. (13)) can thus be written as

$$
\begin{aligned}
\mathrm{d}_{t}\left(\bar{\sigma}_{n}-\bar{\sigma}_{0, i k}\right)= & \\
& {\left[\partial_{t, n}+\partial_{t, \not \chi}+\partial_{t, \dot{\psi}}+\partial_{t, \dot{X}}\right]\left(\bar{\sigma}_{n}-\bar{\sigma}_{0, i k}\right) . }
\end{aligned}
$$

Resonance locking occurs when for a prolonged period of time the right side of Eq. (20) remains almost zero by near cancellation of the first three shift terms $\left(\partial_{t, n}, \partial_{t, \not}\right.$ and $\partial_{t, \dot{x})}$; the last term proportional to $\dot{X}$ is usually much smaller in absolute value than the first three terms. Near the end of core hydrogen burning however, the last term may become important, a situation we have not yet explored.

In a binary system in which the stellar components and the orbit have the same direction of rotation the strongest low order orbital harmonics usually excite retrograde oscillation modes in the stars, spinning the stars down and therefore causing a net positive relative shifting via the term $\partial_{t, \not x}$. In such a case, both factors $\zeta_{i n j}$ and $\mathcal{T}_{i j}^{l m}$ are negative for these low order harmonics $j$. Orbital decay due to dissipation of excited prograde modes in the companion star increases the mean orbital frequency $\omega$ and therefore gives rise to a positive value for the term $\partial_{t, \dot{x}}$ of star $i$. If the self-shift factor $\zeta_{\text {inn }}$ of a nearly resonant higher order prograde harmonic $n$ is negative (for $n$ below the value for which $\zeta$ changes sign, see Eq. (14)) then this harmonic is driven into resonance by the combined effect of the two positive terms $\partial_{t, \not}$ and $\partial_{t, \dot{\psi}}$, whereby its selfshift term $\partial_{t, n}$ becomes increasingly more negative since its (positive) torque $\mathcal{T}_{\text {in }}^{l m}$ becomes larger. Therefore the net relative shifting rate $\mathrm{d}_{t}\left(\bar{\sigma}_{n}-\bar{\sigma}_{0, i k}\right)$, which is the total of all terms, decreases. This results in a longer duration of the resonant excitation and therefore enhances the tidal decay process of the orbit. If the nearly resonantly excited oscillation mode of the star has a sufficiently high resonance peak and the Hansen coefficient of the exciting orbital harmonic $n$ is sufficiently large the self-shift term can counterbalance the rest of the terms in Eq. (20) so that the relative shift rate will drop to zero before the resonance is crossed. During the subsequent period of resonance locking the frequency distance between orbital harmonic $n$ and the resonance usually decreases slowly as the orbital eccentricity declines, because the distribution of the Hansen coefficients for the orbital harmonics will change in favour of the low order harmonics. These excite retrograde modes, requiring the weakening high frequency harmonic $n$ to approach the resonance more closely in order to remain in balance with the other terms in Eq. (20). Eventually, the $n$th orbital harmonic will cross the stellar eigenfrequency, after which the decreasing torque can no longer withstand the driving influence of the other two terms and the locking phase is terminated.

Equilibrium and thus locking of a mode can only be attained if the self-shift term counters the other terms, so for prograde stars $\zeta_{\text {inn }}$ (see Eq. (14)) must be negative. Furthermore, the most effective locking with shortest orbital decay timescales occurs for large torque values $\mathcal{T}_{\text {in }}^{l m}$ and thus, for a given equilibrium value for the self-shift term $\partial_{t, n}$, for small values of $\left|\zeta_{\text {inn }}\right|$ (see Eq. (16)). At the same time, a long duration of locking is achieved when locking is established already while the frequency difference between the harmonic frequency and the eigenfrequency is still relatively large so that the fractional peak height $\mathcal{Q}_{n}$ at which the harmonic excites the oscillation mode is only small. In that case a large torque fraction $\frac{1}{\mathcal{Q}_{n}}$ can yet be gained by further approaching the resonance, so the Hansen coefficient of the exciting harmonic can decrease by a relatively large factor before the locking is terminated. In that case, locking will only end as the eccentricity is significantly decreased.

During locking a second harmonic approaching and possibly crossing another stellar oscillation mode could during a short interval enhance the positive term $\partial_{t, \not x}$, increasing the resonance height $\mathcal{Q}_{n}$ needed to keep the locking balance (20) intact, thereby possibly pushing the 
locked harmonic through resonance and forcing an early end to the equilibrium situation. This can happen when a low order harmonic for which $\zeta<0$ excites a (retrograde) r-mode, or when a high order harmonic for which $\zeta>0$ excites a prograde g-mode. On the other hand, excitation of a prograde g-mode by another harmonic $n_{2}$ for which $\zeta<0$ decreases the term $\partial_{t, \not}$, thereby decreasing the resonance height $\mathcal{Q}_{n}$ of the locked harmonic. If the other harmonic $n_{2}$ quickly crosses its resonance the old locking equilibrium will soon be restored; if, on the other hand, the harmonic $n_{2}$ becomes locked before crossing its resonance, the previously locked harmonic $n$ can be pushed out of resonance, while harmonic $n_{2}$ takes its place as locked harmonic. Likewise, resonance crossings or resonance locking in star 2 will influence the term $\partial_{t, \dot{\psi}}$, thereby influencing the resonance locking mechanism in star 1 .

Obviously resonance locking depends on the ratio $I_{\text {orb }} / I_{i}$, in combination with the order $n$ of the harmonic which is locked. For two $10 M_{\odot}$ stars the orbital moment of inertia is approximately $I_{\text {orb }} \approx 610^{57} P_{\text {orb }}^{\frac{4}{3}} \mathrm{~g} \mathrm{~cm}^{2}$ where $P_{\text {orb }}$ is in days, while the stellar moment of inertia is approximately $I_{i} \approx 210^{56} \mathrm{~g} \mathrm{~cm}^{2}$. For orbits with periods of a few days, therefore, $I_{\text {orb }} \gg I_{i}$. Compared to the case of a $10 M_{\odot}$ MS star and a $1.4 M_{\odot}$ compact companion, as was considered in Paper II, the orbital moment of inertia $I_{\text {orb }}$ at given $P_{\text {orb }}$ is larger by a factor $\frac{100}{20} / \frac{14}{11.4}\left(\frac{20}{11.4}\right)^{\frac{2}{3}} \approx 6$. Likewise, the torque generated by a certain harmonic at a given orbital period (and for equal eccentricities, stellar rotation rates and nuclear evolution stages, compared to the low mass companion case) is larger by a factor $\left(\frac{10}{1.4}\right)^{2}\left(\frac{11.4}{20}\right)^{2} \approx 17$ where the first factor comes from the higher companion mass, and the second factor from the longer semimajor axis for the heavier system.

This increase in torque strength does not change the locking balance much, since the three dominant terms $\partial_{t, n}, \partial_{t, \not \chi}$ and $\partial_{t, \dot{z}}$ in Eq. (20) are all increased by the same factor. However, due to the current large value of $I_{\text {orb }} / I_{i}$ the harmonic $n \simeq m \sqrt{I_{\text {orb }} /\left(3 I_{i}\right)}$ (see Eq. (14)) for which $\zeta_{\text {inn }}$ changes sign is so large that it has too small power to be significant, so that, unlike the cases studied in Paper II, no locking occurs for which $\zeta_{\text {inn }} \simeq 0$. Harmonics up to a higher order will thus counterbalance the positive frequency shifting due to low order retrograde modes, so more high order harmonics can potentially become involved in resonance locking.

In case the direction of the orbital motion of the companion is counter to the direction of stellar rotation only retrograde modes are excited. Hence, resonance locking as a balance between prograde and retrograde harmonics cannot occur. But for significant orbital eccentricity tidal forcing becomes significant at a harmonic $n$ for which the magnitude of $\zeta_{i n n}$ is small and for which $\zeta_{i n n}$ changes sign during the evolution. If this happens, efficient orbital decay may be the result, as was shown in Paper II for the binary radio pulsar system PSR J0045-7319, which was assumed to have significant retrograde stellar rota- tion $\Omega_{\mathrm{s}} \approx 0.3 \Omega_{\mathrm{c}}$ and which has high orbital eccentricity $(e \approx 0.8)$. Since in this case all the torques for the large number of harmonics contributing to Eq. (20) are generated in the region of the strong g-mode resonance peaks and thus fluctuate rapidly in magnitude as evolution proceeds, the locking process is not as stable, and the locked harmonic is seen to erratically move up and down the resonance peak in stead of being driven steadily through resonance. As retrograde rotation is not likely to be encountered in double MS binary systems, we will not consider such systems in this paper.

\section{Results for dual MS binaries}

We now present the results of calculations of the orbital evolution of dual MS binaries for a number of different sets of orbital parameters, and check for the occurrence of resonance locking. For simplicity the same torque spectra are used for the two $10 M_{\odot}$ stars, which have identical values for all other relevant parameters as well. A somewhat artificial situation would occur if both stars at some stage during the orbital evolution would rotate at equal rates. In such a case, the situation would have become fully symmetric between the two stars, so that their evolution would be identical from that point on. In order for our model to resemble more closely the realistic situation in which small differences in mass of the components leads to different evolution for both stars, we lifted the symmetry by simply increasing the rate of stellar evolution of one of the stars by a small amount. We start all our calculations when both stars have a core hydrogen abundance (by mass) of $X_{\mathrm{c}} \simeq 0.4$.

\subsection{Time integration for the secular tidal evolution}

For each timestep the torques acting on the stars are calculated by summing over the harmonic components of the tidal potential, after which the orbital and stellar parameters are updated using a second order Runge-Kutta scheme:

$\boldsymbol{A}\left(t_{i+1}\right)=\boldsymbol{A}\left(t_{i}\right)+\left.\frac{\mathrm{d} \boldsymbol{A}}{\mathrm{d} t}\right|_{t_{i+\frac{1}{2}}}\left(t_{i+1}-t_{i}\right)$

with

$\left.\frac{\mathrm{d} \boldsymbol{A}}{\mathrm{d} t}\right|_{t_{i+\frac{1}{2}}}=\frac{\mathrm{d}}{\mathrm{d} t}\left(\boldsymbol{A}\left(t_{i}\right)+\left.\frac{1}{2} \frac{\mathrm{d} \boldsymbol{A}}{\mathrm{d} t}\right|_{t_{i}}\left(t_{i+1}-t_{i}\right)\right)$

where subscript ${ }_{i}$ counts the timesteps and vector $\boldsymbol{A}=$ $\left(H_{1}, H_{2}, E_{\text {orb }}, e^{2}\right)$. Timesteps are limited by the condition that the relative excitation height of each harmonic should not change by too large a factor from timestep to timestep:

$\frac{1}{1.5} \leq \frac{\mathcal{Q}_{n}\left(t_{i+1}\right)}{\mathcal{Q}_{n}\left(t_{i}\right)} \leq 1.5$

and also by the condition that the difference in excitation height between two timesteps should not exceed $10 \%$ of the total peak height:

$\left|\mathcal{Q}_{n}\left(t_{i+1}\right)-\mathcal{Q}_{n}\left(t_{i}\right)\right| \leq 0.1$. 

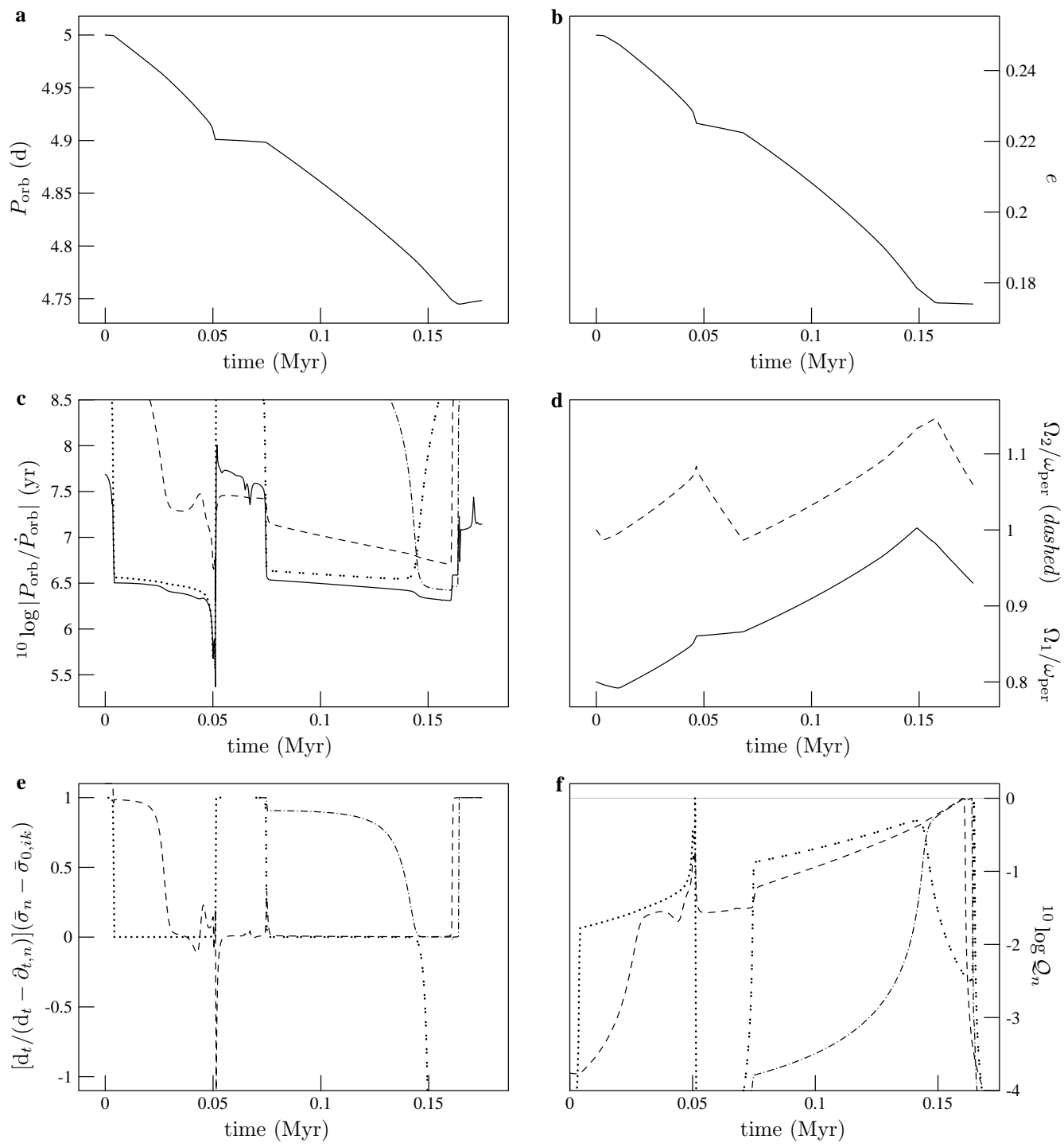

Legend for panels $\boldsymbol{c})$, $\boldsymbol{e}$ ) and $\boldsymbol{f})$ :

$-\cdots n=7, i=1, k=13$

$n=9, i=2, k=10$

...... $n=7, i=2, k=14$

-.... $n=8, i=2, k=11$

Fig. 1. Short interval of orbital evolution for a system containing two $10 M_{\odot}$ MS stars. At $t=0$ the orbit has a period of 5 days (panel a)) and eccentricity $e=0.25$ (panel b)). Initially star 1 rotates at 0.8 times the periastron frequency, star 2 starts (pseudo) synchronously at $\Omega_{2}=\omega_{\text {per }}$ (panel d)). Panel c) gives the tidal evolution timescale, along with partial timescales for the locked harmonics; panel e) indicates the occurrence of resonance locking by giving the shifting rate of locked orbital harmonic frequencies $\bar{\sigma}_{n}$ relative to the locking eigenfrequencies $\bar{\sigma}_{0, i k}$, divided by the sum of all terms contributing to this rate excluding the contribution of harmonic $n$ itself. During resonance locking this value is almost zero. Panel $\mathbf{f}$ ) shows the relative excitation height $\mathcal{Q}_{n}$ of the locked harmonics

Furthermore, to increase the stability of the integration scheme during resonance locking timesteps are chosen such that the second order term is for each timestep much smaller than the first order term:

$$
\begin{aligned}
\epsilon_{j}(O(2)) \equiv \frac{1}{A_{j}\left(t_{i+1}\right)}\left(A_{j}\left(t_{i}\right)\right. & +\left.\frac{\mathrm{d} A_{j}}{\mathrm{~d} t}\right|_{t_{i}}\left(t_{i+1}-t_{i}\right) \\
& \left.-A_{j}\left(t_{i+1}\right)\right) \lesssim 10^{-7}
\end{aligned}
$$

for each component $j$ of the vector $\boldsymbol{A}$.

\subsection{Simultaneous resonance locking: a detailed description}

Figure 1 depicts a short interval of orbital evolution as an example of resonance locking taking place in both stars simultaneously. The initial orbit has a period $P_{\text {orb }}=5$ days and eccentricity $e=0.25$. For this moderate orbital eccentricity only orbital harmonics with $n<10$ have to be considered. Starting with star 1 rotating at $0.8 \omega_{\text {per }}$ and star 2 rotating at periastron frequency $\left(\omega_{\text {per }}=\sqrt{\frac{1+e}{(1-e)^{3}}} \omega\right)$, panels $1 \mathrm{a}$ and $1 \mathrm{~b}$ show the evolution of orbital parameters, while panel $1 \mathrm{~d}$ gives the rotation rates of the stars. 
As the evolution progresses, we observe intervals during which the orbit declines rapidly and the tidal timescale (solid line in panel 1c) stays at a more or less constant low level, reminiscent of the resonance locking described in Paper II. In panel 1c partial timescales which can be formed from the torques that separate harmonic components $n$ of the tidal potential exert on the stars $i$ are also given: $\tau_{i n}=\frac{P_{\text {orb }}}{\dot{P}_{\text {orb }, i n}}=\frac{2}{3} \frac{a}{\dot{a}_{\text {in }}}=-\frac{2}{3} \frac{E_{\text {orb }}}{E_{\text {orb }, i n}}$ where, according to Eq. (4), $\dot{E}_{\text {orb }, i n}=n \omega \mathcal{T}_{i n}^{l m}$. For the harmonics that have the strongest torque contributions and therefore the shortest partial orbital decay timescales panel 1e confirms resonance locking by showing that the rate $\mathrm{d}_{t}\left(\bar{\sigma}_{n}-\bar{\sigma}_{0, i k}\right)$ at which these harmonic frequencies $\bar{\sigma}_{n}$ drift in frequency relative to their resonant stellar oscillation frequency $\bar{\sigma}_{0, i k}$ almost vanishes due to the action of their self-shift term $\partial_{t, n}\left(\bar{\sigma}_{n}-\bar{\sigma}_{0, i k}\right)$. The quantity plotted in panel 1e,

$$
\begin{aligned}
& {\left[\frac{\mathrm{d}_{t}}{\mathrm{~d}_{t}-\partial_{t, n}}\right]\left(\bar{\sigma}_{n}-\bar{\sigma}_{0, i k}\right) \equiv} \\
& {\left[\frac{\partial_{t, n}+\partial_{t, \not x}+\partial_{t, \dot{\not}}+\partial_{t, \dot{X}}}{\partial_{t, \not x}+\partial_{t, \dot{\not}}+\partial_{t, \dot{X}}}\right]\left(\bar{\sigma}_{n}-\bar{\sigma}_{0, i k}\right),}
\end{aligned}
$$

is close to unity when $\bar{\sigma}_{n}$ is far from the resonance frequency $\bar{\sigma}_{0, i k}$ so that $\partial_{t, n}\left(\bar{\sigma}_{n}-\bar{\sigma}_{0, i k}\right)=\zeta_{\text {inn }} \mathcal{T}_{i n}^{l m}$ is small. During resonance locking $\partial_{t, n}$ cancels $\partial_{t, \not x}+\partial_{t, \dot{\not}}+\partial_{t, \dot{X}}$, so that the numerator in Eq. (21) vanishes and the corresponding line in panel 1e falls off to zero. Figure $3 \mathrm{f}$ gives the fraction $\mathcal{Q}_{n}$ of the total height of the resonance peak at which the locked harmonics excite their resonant stellar modes.

Figure 2 shows the position of the stellar eigenfrequencies $\sigma_{0, i k}$ (solid lines) relative to the harmonics of the orbital frequency $\sigma_{n}$ (dashed lines) in the inertial frame; panel $2 \mathrm{a}$ for star 1 and panel $2 \mathrm{~b}$ for star 2 . The positions of the stellar eigenfrequencies have thus been corrected for the stellar spin frequency: $\sigma_{0, i k}=\bar{\sigma}_{0, i k}+m \Omega_{i}$. The frequencies have been normalised by the mean orbital frequency $\omega$ which itself changes (and increases most of the time) during the evolution (see Fig. 1a). In this way the orbital harmonics $\sigma_{n} / \omega=n$ are located at the natural numbers and show up as horizontal (dashed) lines in the graph. The relative magnitude of the harmonic potential terms is indicated by the greyscale which reflects the strength of the Hansen coefficients $h_{n}$. The long dashed line gives the dimensionless frequency for which $\bar{\sigma}=0$, so harmonics which are located above this line excite prograde modes in the star, while harmonics below this line are retrograde. The thick line segments indicate resonance locking for those harmonics $n$ which meet with the condition that the drift of the harmonic relative to the resonance frequency almost cancels:

$\left|\left[\frac{\mathrm{d}_{t}}{\mathrm{~d}_{t}-\partial_{t, n}}\right]\left(\bar{\sigma}_{n}-\bar{\sigma}_{0, i k}\right)\right| \leq 0.05$,

and which have a fractional excitation height of at least $1 \%$ :

$\mathcal{Q}_{n} \geq 0.01$ so only resonance locking which may significantly enhance the orbital decay will show up. In Fig. 2 the prograde gmodes $\mathrm{g}_{10}^{2}$ and $\mathrm{g}_{20}^{2}$ are labelled, while the range in which the retrograde $\mathrm{r}$-modes are located is labelled $r_{k}$.

Figure 3 shows for the locking which occurs in the two stars the separate terms of the locking balance Eq. (20). Locking of harmonic $n=7$ on the stellar mode $\mathrm{g}_{13}^{2}$ of star 1 is shown in panel 3a, while in panel 3b locking of harmonic $n=9$ onto mode $\mathrm{g}_{10}^{2}$ of star 2 is followed by the combined locking of $n=7$ and 8 locking respectively on the modes $\mathrm{g}_{14}^{2}$ and $\mathrm{g}_{11}^{2}$ of star 2 . Here, thick lines are used to distinguish the $n=8$ locking from the locking of $n=7$. The solid lines which give the net shifting rates $\mathrm{d}_{t}\left(\bar{\sigma}_{n}-\right.$ $\left.\bar{\sigma}_{0, i k}\right)$ drop to zero during resonance locking due to the fact that the level of the dashed lines which give the selfshifting rates $\partial_{t, n}\left(\bar{\sigma}_{n}-\bar{\sigma}_{0, i k}\right)$ cancels the combined action of the dash-dotted and dotted lines, which stand for the terms $\partial_{t, \not}\left(\bar{\sigma}_{n}-\bar{\sigma}_{0, i k}\right)$ and $\partial_{t, i}\left(\bar{\sigma}_{n}-\bar{\sigma}_{0, i k}\right)$, respectively. The stellar evolution term $\partial_{t, \dot{X}}\left(\bar{\sigma}_{n}-\bar{\sigma}_{0, i k}\right)$ is fairly small and almost constant, and therefore not shown as a separate line in the plots. Note that resonance locking in one star can only couple to the other star through its shrinking or expansion effect on the orbit, which is given by the dotted lines in Fig. 3.

The dominant orbital harmonic with frequency $\bar{\sigma}_{2}$ excites retrograde modes in both stars. However, the second strongest harmonic frequency $\bar{\sigma}_{3}$ is prograde in star 1 and retrograde in star 2, see Fig. 2. As a consequence, the dash-dotted lines in Fig. 3 which are constructed from the sum of all non-locked harmonics correspond to more positive values for star 2 (panel 3b) than for star 1 (panel 3a). At $t=0$ the level of the dash-dotted line in star 1 is even almost zero due to the action of prograde harmonic $n=10$ which slowly approaches the $g_{7}^{2}$ resonance peak of star 1, and which crosses the peak of the resonance near $t=0.044$ Myr. Soon after the evolution started, $\bar{\sigma}_{9}$ encounters $\mathrm{g}_{10}^{2}$ of star 2 , and as it approaches the resonance (panel 1f), its self-shift term becomes strongly negative, causing its net shift rate to slow down (respectively dashed and solid lines in panel $3 \mathrm{~b}$ ). When its net shift rate (solid line) reaches zero, resonance locking is established and from that moment on its approach of the resonance frequency $\bar{\sigma}_{0,210}$ (see dotted line panel 1f) happens on the timescale at which significant eccentricity decrease occurs (see panel 1b). When the eccentricity declines the strength of the Hansen coefficient $h_{9}$ is reduced and, to maintain the locking equilibrium, this has to be compensated by a closer approach of the resonance. This can be seen in panel $1 \mathrm{f}$ which shows the evolution of the relative resonance height $\mathcal{Q}_{9}$.

Due to the $n=9$ resonance locking in star 2 and the resulting orbital shrinking, the rate at which $\bar{\sigma}_{7}$ approaches $\mathrm{g}_{13}^{2}$ in star 1 increases (dotted and solid lines in panel 3a). As the relative height $\mathcal{Q}_{7}$ in the peak of $\mathrm{g}_{13}^{2}$ of star 1 increases (dashed line panel 1f), its counteracting self-shift term becomes more strongly negative (dashed line panel 3a) and after approximately 0.03 Myr harmonic $n=7$ becomes locked as well. The above 

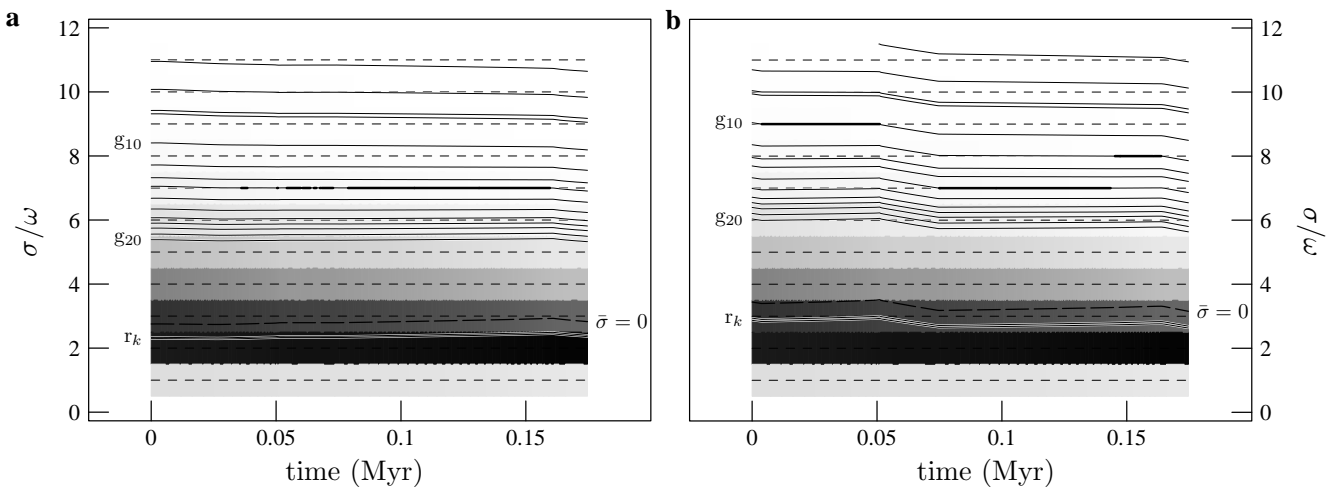

Fig. 2. The curves show, for the orbital evolution pictured in Fig. 1, the stellar eigenfrequencies in the inertial frame $\sigma_{0, i k}$ of star 1 (panel a)) and star 2 (panel b)) normalised on the varying mean orbital frequency $\omega$. A resonance occurs when a curve coincides with one of the horizontal dashed lines (which correspond to the orbital harmonics $n=\sigma_{n} / \omega$ ). The boundary between pro- and retrograde frequencies $(\bar{\sigma}=0)$ is given by the long fat dashes, and the relative strength of the different orbital harmonic components given by the Hansen coefficients $h_{n}$ is coded in greyscale. Thick line segments on the curves mark resonance locking. The r-modes occupy a narrow frequency region indicated by $r_{k}$
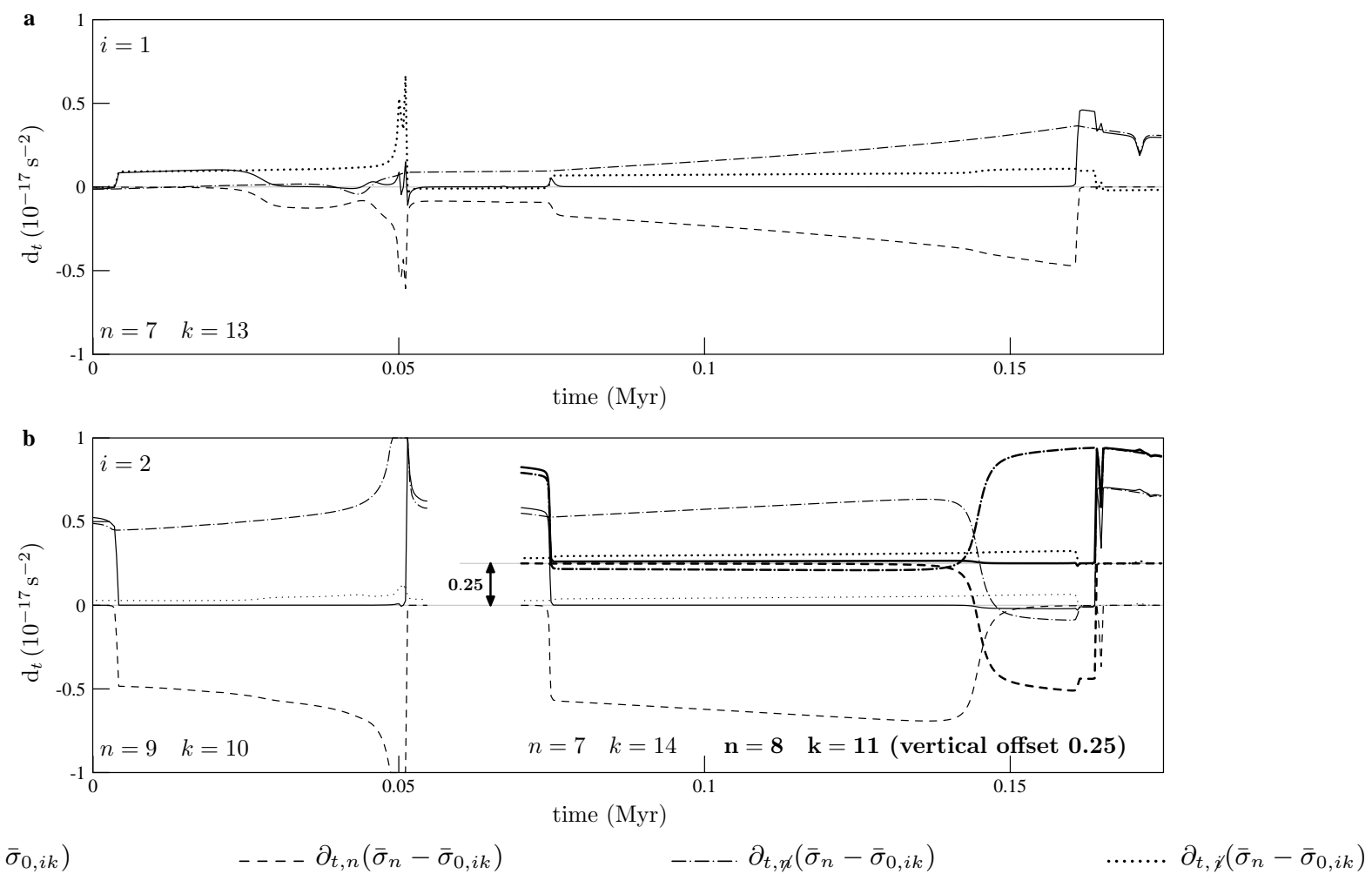

$$
\begin{aligned}
& \mathrm{d}_{t}\left(\bar{\sigma}_{n}-\bar{\sigma}_{0, i k}\right) \\
& \ldots \partial_{t, \not x}\left(\bar{\sigma}_{n}-\bar{\sigma}_{0, i k}\right) \\
& \partial_{t, i}\left(\bar{\sigma}_{n}-\bar{\sigma}_{0, i k}\right)
\end{aligned}
$$

Fig. 3. The resonance locking balance of star 1 (panel a)) and star 2 (panel b)) during the orbital evolution depicted in Fig. 1. The shifting rate of each locked harmonic relative to the eigenfrequency of the mode it is locked to and the separate terms which contribute to this rate are plotted, see Eq. (20). Not shown is $\partial_{t, \dot{X}}\left(\bar{\sigma}_{n}-\bar{\sigma}_{0, i k}\right)$, which is small and almost constant. To prevent over-plotting a vertical offset has been applied for the case $n=8 k=11$ (thick lines)

mentioned resonance crossing of $\bar{\sigma}_{10}$ through $\mathrm{g}_{7}^{2}$ of star 1 which quickly follows causes the dash-dotted line in panel 3 a to temporarily take on a negative value and, as a reaction, the resonance height $\mathcal{Q}_{7}$ in star 1 decreases for a while (panel 1f).

Next, just before $t=0.05 \mathrm{Myr}$, orbital harmonic $n=3$ passes twice through the weakest $\mathrm{r}$-mode that is included in the spectrum of star 2, first downwards and upwards quickly after that. The strong r-mode resonances spin the star down and widen the orbit, inducing a positive frequency shift in the locking balance. Primarily these rmode crossings in star 2 feed into the dash-dotted line in panel $3 \mathrm{~b}$, driving the $n=9$ harmonic deeper into resonance. Thereby the self-shift term $\partial_{t, 9}\left(\bar{\sigma}_{9}-\bar{\sigma}_{0,210}\right)$ (dashed line in $3 \mathrm{~b}$ ) has to become more negative in order to keep the locking equilibrium intact. The orbital expansion shows up as a rising of the dotted line in $3 \mathrm{a}$, and this in turn drives the $n=7$ locking balance in star 1 deeper 
into resonance (dashed lines panels $1 \mathrm{f}$ and $3 \mathrm{a}$ ). A little later at $t \simeq 0.05 \mathrm{Myr}$, during the second $\mathrm{r}$-mode crossing in star 2 , the $n=9$ locking in star 2 terminates when this harmonic is driven over the peak of the resonance.

Using the orbital evolution described up to this point a few remarks can be made about the way resonance lockings and resonance crossings in the two stars can influence each other. As already mentioned, resonance locking in one star can indeed through orbital changes cause resonance locking in the other star to become more intense. Note the enhanced positive levels of the dotted lines in Fig. 3 which must be countered by the negative self-shift terms, so the levels of the dashed lines must become more negative by raising the fractional resonance heights $\mathcal{Q}_{n}$ of the locked harmonics. When locking in star 2 commences, the rate at which harmonic $n=7$ approaches resonance with the $\mathrm{g}_{13}^{2}$ mode of star 1 it will become locked on increases strongly. Resonance locking in star 1 would therefore not have occurred as quickly if no resonance locking in star 2 had occurred. At the beginning of resonance locking in star 1 , the shrinking of the orbit due to locking in star 2 , shown by the dotted line in panel $3 \mathrm{a}$, is even the strongest positive term against which the $n=7$ harmonic becomes locked in star 1 . Definitely, no locking would have occurred here without the locking in star 2.

A resonance crossing of an $\mathrm{r}$-mode in star 2 terminates the locking in star 2 , because the resonance peak on which the $n=9$ harmonic had been locked could not provide sufficiently large a torque to keep the resonance locking balance (20) in star 2 near zero. The r-modes' indirect influence (via stellar spin down and orbital expansion) on the resonance locking in star 1 is also large; the required fractional peak height of $\bar{\sigma}_{7}$ in star 1 (dashed line panel 1f) increases to more than $10 \%$. A stronger locked mode in star 2 could have prevented locking in star 2 to terminate, but it could also have caused the locking in star 1 to end if the resonant torque required to maintain locking in star 1 would have increased above the maximum of the locked mode in star 1 . Instead, locking in star 1 continues after the locking in star 2 has ended around $t=0.05 \mathrm{Myr}$. As $\bar{\sigma}_{10}$, which crossed through $\mathrm{g}_{7}^{2}$ of star 1 , drifts further away from resonance its influence on the level of the dash-dotted line in panel 3 a subsides, and the level of this line rises due to the retrograde excitation of $\bar{\sigma}_{2}$ to become the largest positive term in the resonance locking balance of star 1 . Meanwhile, the lack of resonant prograde forcing in star 2 to counter the retrograde torques due to the leading harmonic terms $n=2$ and $n=3$ causes this star to rapidly spin down after $t \simeq 0.05 \mathrm{Myr}$ (dashed line panel $1 \mathrm{~d}$ ) to near pseudo-synchronisation. As a consequence, spin energy is transferred from star 2 into the orbit while simultaneously, due to the continuing locking in star 1, energy is being lost from the orbit at a higher rate. During the time interval in which no locking in star 2 occurs, the partial tidal timescale associated with harmonic $n=7$ acting on star 1 is shorter than the total tidal timescale for the system (dashed and solid lines in panel 1c). The rapid spin down of star 2 induces a quick drift of its oscillation fre- quencies relative to the harmonic frequencies of the orbit, see panel $2 \mathrm{~b}$.

Around $t=0.075 \mathrm{Myr} \bar{\sigma}_{7}$ runs into and becomes locked on $\mathrm{g}_{14}^{2}$ of star 2 , so that the orbital decay is enhanced. This causes the dotted line in panel 3 to rise and forces the resonance locking in star 1 to readjust, whereby the fractional resonance height of the $(n=7)$ locked mode in star 1 increases somewhat (dashed line panel 1f). In turn this rises the level of the dotted line in panel $3 \mathrm{~b}$ somewhat and causes the locking in star 2 to intensify, but this effect is only small. During locking the harmonic frequency $\bar{\sigma}_{7}$ approaches the eigenfrequency $\bar{\sigma}_{0,214}$ and its fractional excitation height rises. However the relative peak height of $\bar{\sigma}_{8}$ which approaches $\bar{\sigma}_{0,211}$ increases faster, see the double dotted and dash-dotted lines in panel 1f. As the relative peak height of $\bar{\sigma}_{8}$ increases its self-shift term becomes more negative (thick dashed line in panel $3 \mathrm{~b}$ ), thereby decreasing the term $\partial_{t, \not x}\left(\bar{\sigma}_{7}-\bar{\sigma}_{0,214}\right)$ (thin dash-dotted line in panel $3 \mathrm{~b}$ ) of which it is a part along with the terms due to the strong low order orbital harmonics. In reaction the self-shift term of the $n=7$ locking in star 2 must become less negative (thin dashed line in panel $3 \mathrm{~b}$ ), so the harmonic excitation frequency $\bar{\sigma}_{7}$ drifts away from the resonance frequency, thus lowering the fractional excitation height (double dots in panel 1f). Locking of the $n=8$ harmonic on $\mathrm{g}_{11}^{2}$ then takes the place of the $n=7$ locking on $\mathrm{g}_{14}^{2}$ in star 2 . This $n=8$ locking ends when $\mathcal{Q}_{8}$ reaches 1 (dash-dotted line in panel 1f) and the resonance is crossed, but not before the same happens for $\bar{\sigma}_{7}$ in star 1 . When $\bar{\sigma}_{8}$ crosses the resonance in star 2 its diminishing effect on the term $\partial_{t, \not x}\left(\bar{\sigma}_{7}-\bar{\sigma}_{0,214}\right)$ abruptly ends, and as a consequence $\bar{\sigma}_{7}$ rapidly approaches $\bar{\sigma}_{0,214}$ again. In principle its resonance locking could be reestablished at that point, however the decreasing eccentricity caused the Hansen coefficient $h_{7}$ to become weaker, and the resonant torque needed to balance the locking Eq. (20) for the $n=7$ harmonic cannot be reached by the $k=14$ resonance. Soon after, $\bar{\sigma}_{7}$ also crosses resonance.

From the above detailed description of a short evolution timespan it is clear that resonance locking in one of the early type stars in double MS binaries can establish and/or intensify the locking process in the other component. However, locking in each of the components as well as non-locked resonance crossings can in fact also shorten the period of locking in the other component. The effectiveness of resonance locking as a mechanism for tidal evolution then depends on what the chances are of quickly running into the next stage of locking. To gain some insight in this matter, we will now consider somewhat wider systems and follow their evolution during longer timespans.

\subsection{Occurrence of resonance locking in wider orbits}

For a few increasingly wider initial orbits we follow the orbital evolution and show how resonance locking influences the orbital evolution. In this way we acquire a feeling for 

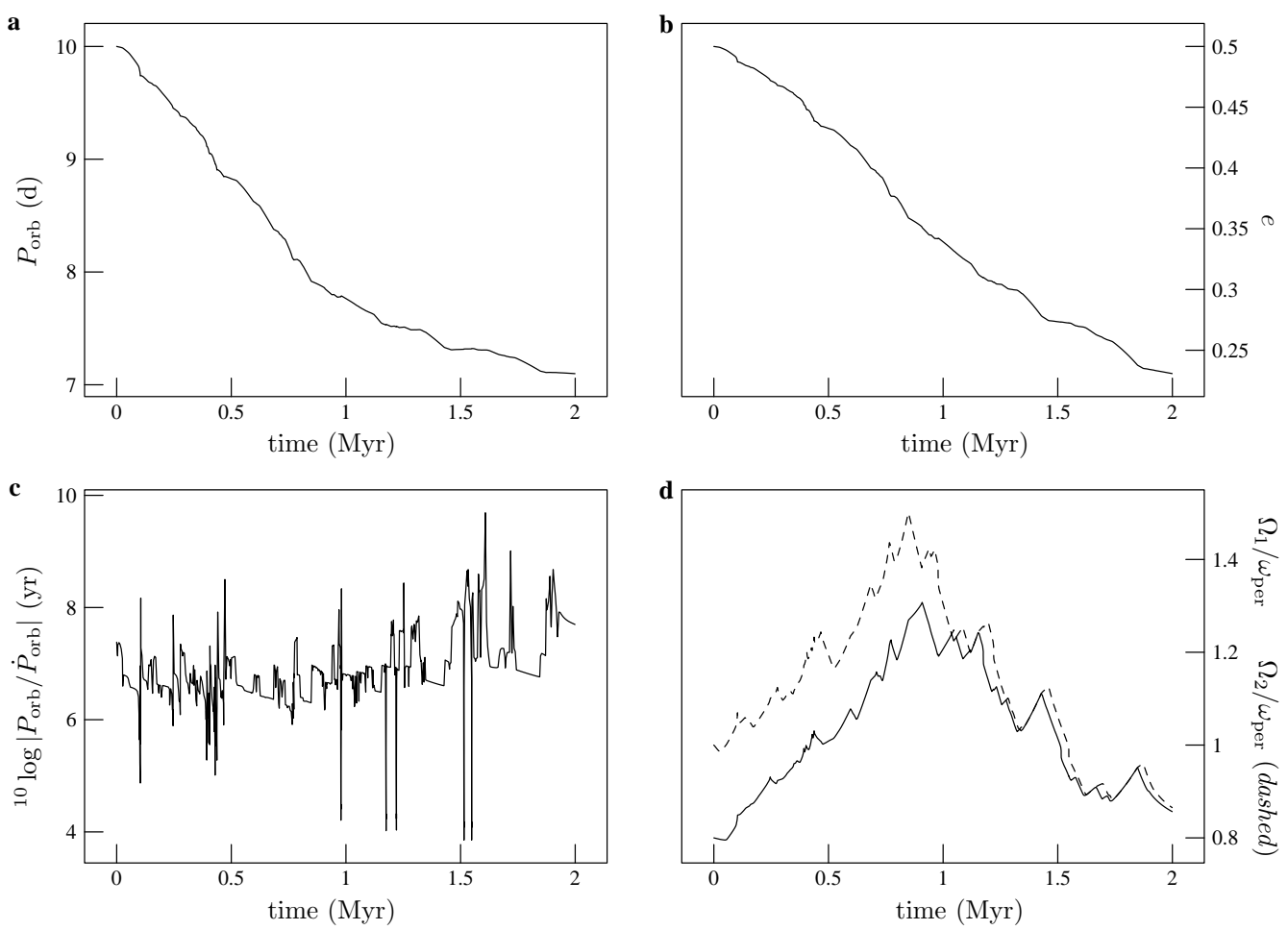

Fig. 4. At $t=0$ the orbit has a period $P_{\text {orb }}=10$ days (panel a)) and eccentricity $e=0.5$ (panel b)). The stars rotate initially at $80 \%$ and $100 \%$ of periastron frequency (panel d)). Resonance locking causes many periods of rapid orbital decay (square dips in panel c)), during which the locked star(s) spins up

the importance of the resonance locking mechanism for a range of different orbital parameters.

\subsubsection{Orbit with $P_{\text {orb }}=10$ days}

First we evolve a somewhat wider orbit $\left(P_{\text {orb }}=10 \mathrm{~d}\right)$, starting from eccentricity $e=0.5$ and follow it for some 2 Myr (see Fig. 4). The stellar rotation rates (panel 4d) at $t=0$ are again $80 \%$ and $100 \%$ of periastron frequency. The timescale for orbital decay (panel 4c) shows many periods during which the orbit decays quite rapidly for tens of thousands of years. During these periods one or both stars have their rotation rate increased. Often the resonance locking occurring during these phases increases the rate of orbital decay by a large fraction, as can be seen from the jumps in the timescale as these resonance locking phases commence or end.

Resonance locking occurs in both stars throughout the evolution calculated here, see Fig. 5. The dotted line in this figure gives the dimensionless frequency for which the self-shift term would exactly vanish:

$\zeta_{\text {inn }}=0 \Rightarrow n=\sqrt{\frac{I_{\text {orb }}}{I_{i}} \frac{m}{3}\left(m+\frac{\partial \bar{\sigma}_{0, i k}}{\partial \Omega_{i}}\right)}$.

As the term $\frac{\partial \bar{\sigma}_{0, i k}}{\partial \Omega_{i}}$ is different for the different oscillation modes, a slight jump in the dotted line is seen each time locking on another stellar g-mode commences. Harmonics of order $n$ lower than this but with $n>n_{0}$ (where $n_{0}$ defines the boundary between prograde and retrograde modes) have negative self-shift and may therefore be locked. The conditions for resonance locking are most favourable in a frequency strip around $n \approx 15$ at first, decreasing towards $n \approx 9$ as the orbital period and eccentricity diminish and the high order Hansen coefficients become much weaker than the Hansen coefficients of the low order retrograde harmonics. During the later stages, significant resonance locking only occurs with the $\mathrm{g}_{14}^{2}$ modes of the stars, which can generate a significantly higher torque value at the top of the resonance peak than the $\mathrm{g}_{15}^{2}$ modes or those with still higher radial orders. At higher frequencies the orbital harmonics are too weak to excite the (weakly damped) modes sufficiently to establish locking, while towards the lower frequencies the stellar gmodes are too heavily damped and generate too small intrinsic torques.

Some of the square dips in the timescale in panel $4 \mathrm{c}$ end in a sharp dip. Similar to what was seen in Sect. 3.2, resonance crossing of a low order harmonic through an r-mode is in these cases responsible for disturbing the resonance locking. The dimensionless $\mathrm{r}$-mode frequencies $\sigma_{0, i k}=\bar{\sigma}_{0, i k}+m \Omega_{i}$ (in the inertial frame) are increased by the locking of prograde high order harmonics (by which the star is spun up), which during the first Myr causes the r-modes to approach their nearest harmonic frequencies $(n=5$ in star 1 and $n=6$ in star 2) many times (see Fig. 5). However, every time the r-mode excitation terminates the locking responsible for the stellar spinup by pushing the locked high order harmonic through resonance, preventing resonance crossing of the strong 

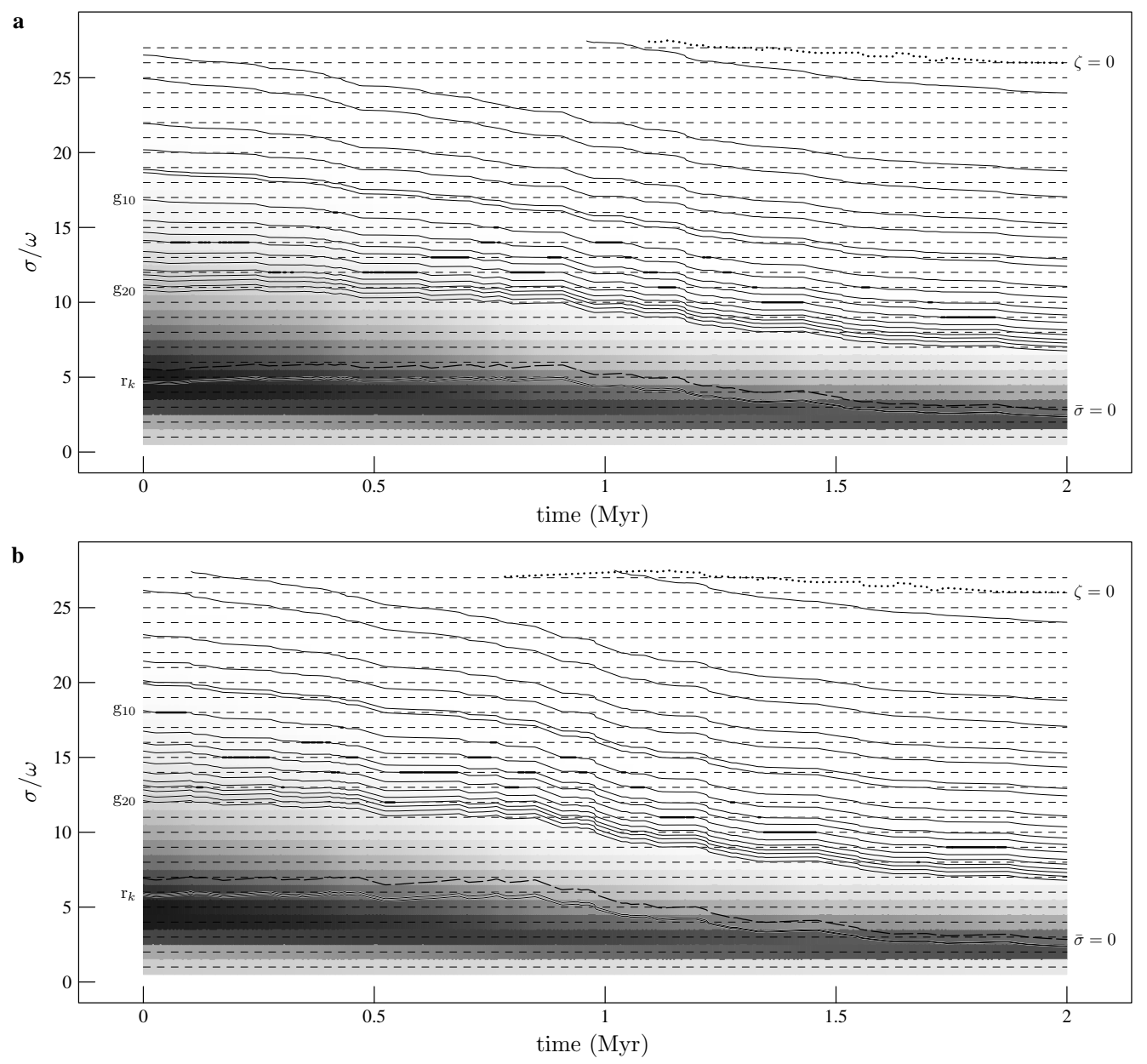

Fig. 5. For the orbital evolution pictured in Fig. 4 (initially $P_{\mathrm{orb}}=10 \mathrm{~d}, e=0.5$ ), the solid curves show the stellar eigenfrequencies $\sigma_{0, i k}$ of star 1 (panel a)) and star 2 (panel b)) normalised on the varying orbital frequency $\omega$. The normalised harmonic frequencies $\sigma_{n} / \omega=n$ of the orbit are plotted as horizontal dashed lines. The boundary between pro- and retrograde frequencies $(\bar{\sigma}=0)$ is given by the long dashes, and the relative strength of the different orbital harmonic components given by the Hansen coefficients $h_{n}$ is coded in greyscale. Thick line segments mark resonance locking; the dotted line gives for the locking oscillation mode the normalised frequency for which $\zeta=0$. Resonance locking in at least one star is established during $64 \%$ of the time

r-modes. Once the next locking of a high order harmonic is established, the r-modes again close in on the nearby orbital harmonic frequency, again putting an early end to the locking at high frequency. As a result, periods of spin-up due to resonance locking of high frequency orbital harmonics and periods of spin-down due to the retrograde forcing of low order orbital harmonics combined with the intrinsic spin-down due to stellar expansion alternate quickly during the first Myr, the first effect dominating during these early stages. Panel $4 \mathrm{~d}$ shows that during the first half of the orbital evolution the stellar rotation speeds increase to supersynchronous rates.

In some of the cases resonance locking of a mode ends not because of r-mode actions, but exactly when resonance locking of a second prograde mode in the same star begins. For these cases the same mechanism applies which appeared during the second half of the evolution described in Sect. 3.2 in star 2, in which resonance locking of one harmonic makes place for locking of another harmonic. The harmonic that becomes locked initially is pushed back out of resonance due to the spin up effect of the other harmonic which approaches its resonance peak faster, and which becomes locked thereafter. As the evolution progresses and the eccentricity decreases the locked second harmonic slowly approaches and eventually crosses its locked resonance frequency. After that, the harmonic that became locked first is no longer held out of resonance, and it quickly re-approaches the mode it was locked to and crosses the resonance.

During this first interval the eccentricity decreases from 0.5 to approximately 0.4 , which causes a significant change in the Hansen distribution of orbital harmonics. The peak of this distribution moves to a lower order harmonic (from $n=5$ to $n=3$ ) and the high order harmonics become much weaker (e.g. a factor 11 for $h_{15}$ ). As a consequence resonance locking of the higher order harmonics becomes less efficient, which is reflected in the fact that 

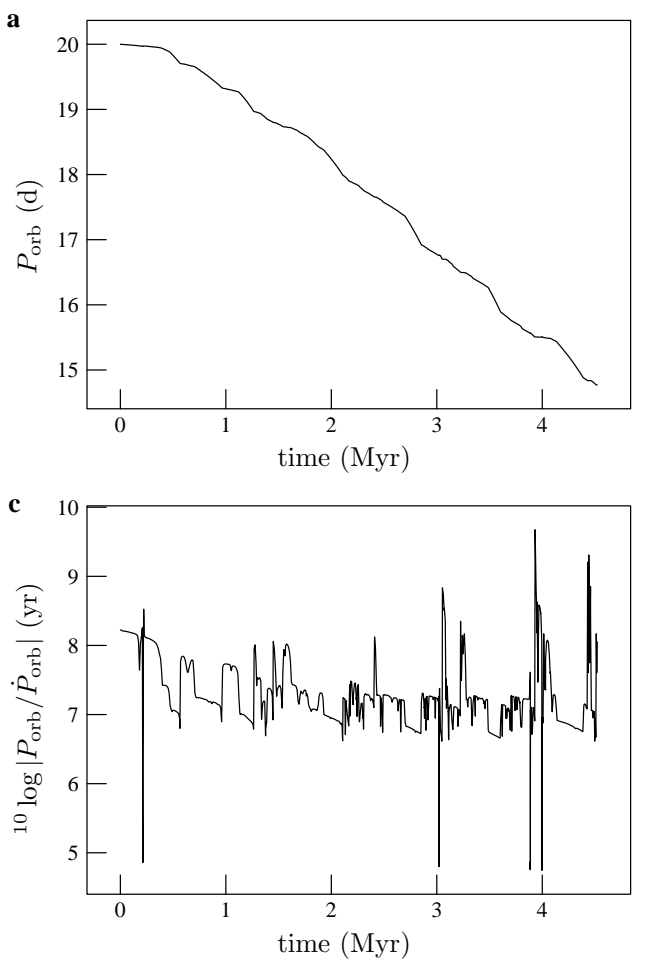
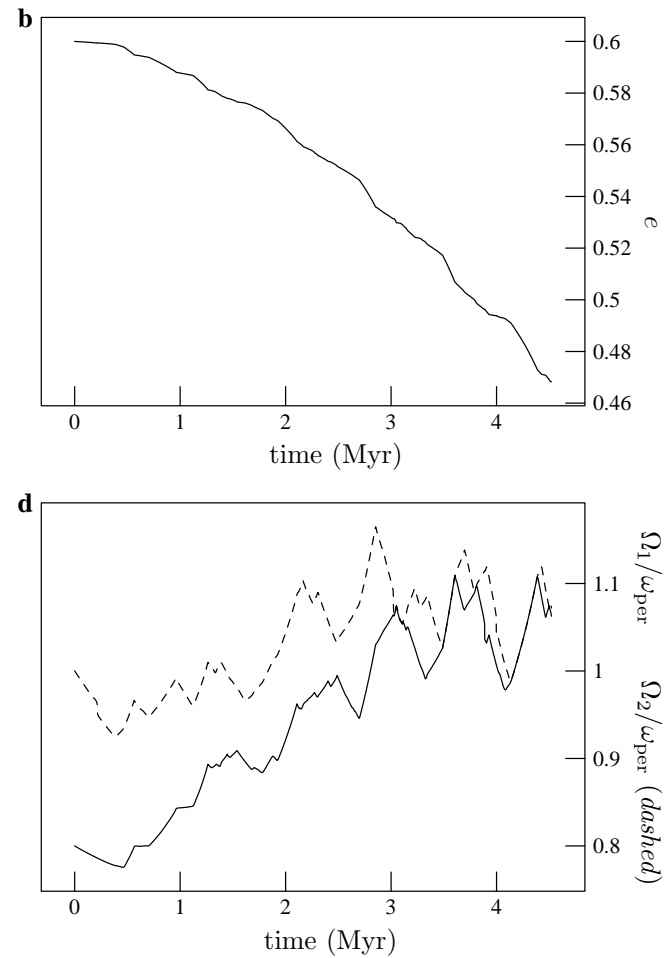

Fig. 6. At $t=0$ the orbit has period $P_{\text {orb }}=20$ days (panel a)) and eccentricity $e=0.6$ (panel b)), and the stars are rotating at $80 \%$ and $100 \%$ of periastron frequency (panel d)). As in Fig. 4, resonance locking causes many periods of rapid orbital decay (square dips in panel c))

the tidal timescale during locking (square dips in panel 4c) increases as the orbit becomes more circularized.

After approximately 1 Myr the resonant spin up during locking is on average no longer dominant over the stellar spin down caused by the low order retrograde harmonics, and therefore the stars show a net spin down (see panel 4d) whereby the r-modes drift downward in Fig. 5. During the second half of the evolution resonance locking is not terminated due to the action of r-modes, but rather because of the decreasing Hansen coefficients of the locked harmonics. Locking during this stage mainly occurs on the relatively strong $\mathrm{g}_{14}^{2}$ modes in both stars, whose oscillation frequencies move downwards in Fig. 5, pausing at each harmonic (dashed line) until the harmonic becomes too weak, and continuing the descent thereafter. The ratio between the resonant spin up effects of the high order harmonics and the spin down effects of the low harmonic order retrograde forcing being the same for both stars, the stellar spin rates during these late stages remain approximately equal. The reason that the initial difference in rotation rates of the stars stays intact during the first half of the evolution is that the stellar spin up and spin down periods are not yet balanced; rather the dominating spin up is sporadically interrupted due to r-mode excitations. Since r-mode excitations during the early stages occur equally often in both stars, the occurrence (and frequency of termination) of resonance locking is approximately equal in both stars, leading to approximately equal average spin-up rates and therefore conservation of the ini- tial difference in rotation speeds. During the second half of the evolution resonance locking is terminated because the higher order locked harmonics weaken as the eccentricity decreases. Initially star 2 is rotating faster, implying its eigenfrequencies in the inertial frame correspond to higher and therefore weaker orbital harmonics. As a result resonance locking for this fastest rotating star will on average last shorter than resonance locking in the slower rotator, and as a consequence the fastest star will on average spin down fastest. Eventually, the rotation rates of the stars become approximately equal.

Though the weakening resonance locking cannot prevent the stars from becoming sub-synchronous rotators near the end, the effect of resonance locking on the orbital evolution remains large during these late stages. Holding a ruler vertically and running it horizontally along the time axis of Fig. 5 one observes that usually at least one of both stars will at any time experience resonance locking. Also resonance locking in both stars is seen to coexist for long periods of time, especially near the end of the evolution where the stars have reached similar rotation speeds. Given the fact that resonance locking occurs very frequently, and that orbital decay during locking is many times stronger than in the non-locked case, we can conclude that resonance locking of relatively weak high frequency orbital harmonics is the most important mechanism for tidal decay during the orbital evolution with $P_{\text {orb }} \lesssim 10 \mathrm{~d}$ shown here. The accumulated time during which at least one star is locked in resonance spans $64 \%$ of 

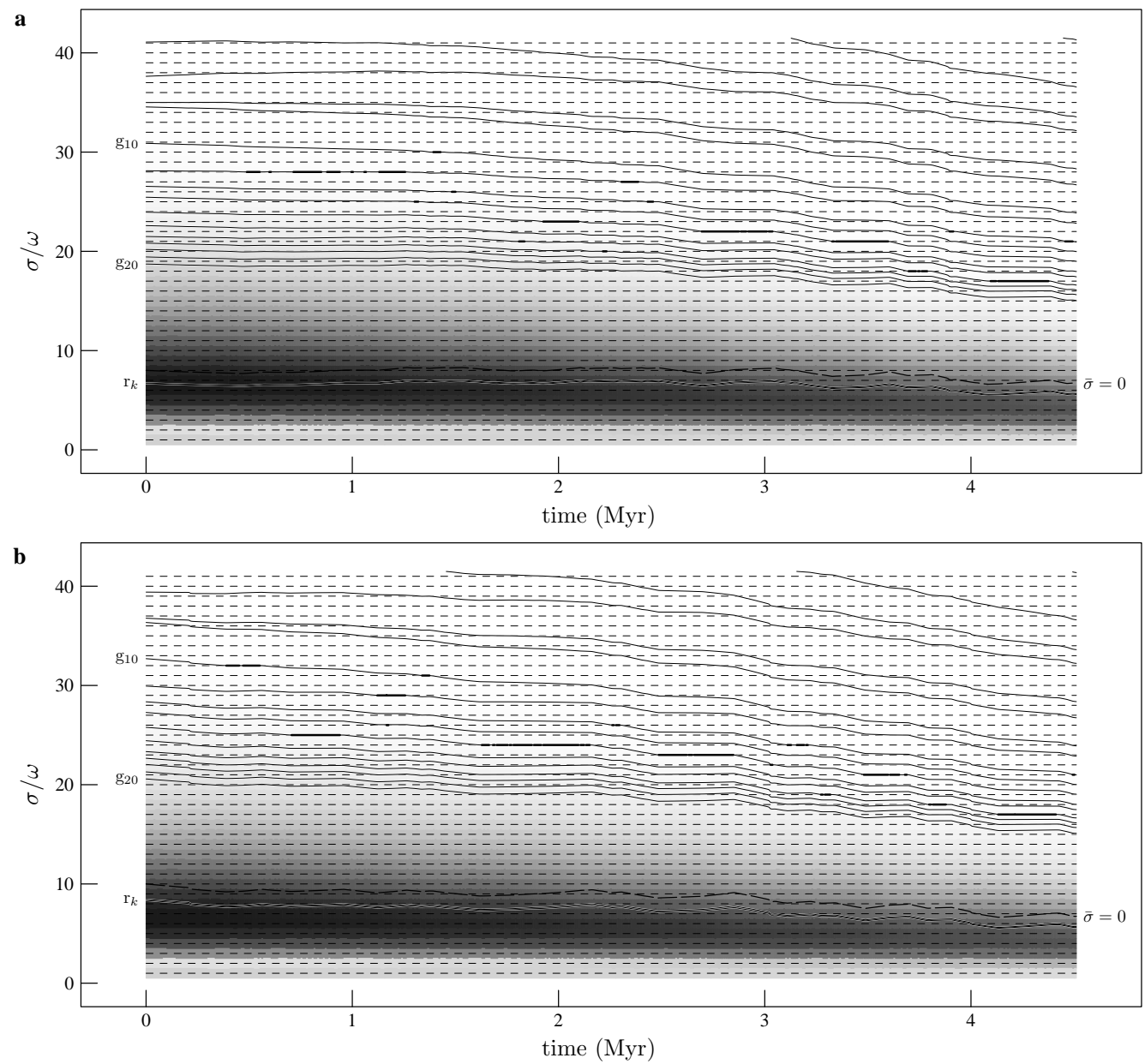

Fig. 7. For the orbital evolution pictured in Fig. 6 (initially $P_{\mathrm{orb}}=20 \mathrm{~d}, e=0.6$ ), the solid curves show the stellar eigenfrequencies $\sigma_{0, i k}$ of star 1 (panel a)) and star 2 (panel b)) normalised on the varying orbital frequency $\omega$. The normalised harmonic frequencies $\sigma_{n} / \omega=n$ of the orbit are plotted as horizontal dashed lines. The boundary between pro- and retrograde frequencies $(\bar{\sigma}=0)$ is given by the long dashes, and the relative strength of the different orbital harmonic components given by the Hansen coefficients $h_{n}$ is coded in greyscale. Thick lines mark resonance locking; the normalised frequency for which $\zeta=0$ for the locked mode is too high for the scale of this plot and cannot be shown. Resonance locking in at least one star is established during $59 \%$ of the time

the total evolution time, while $81 \%$ of the total eccentricity decrease is accounted for during the locking periods.

\subsubsection{Orbit with $P_{\text {orb }}=20$ days}

We now turn our attention to a system with a wider orbit and higher eccentricity. Figure 6 shows the evolution of a system starting at $P_{\text {orb }}=20$ days and eccentricity $e=0.6$, and we evolve the binary system for some 4.5 Myr until the central hydrogen abundance of one of the components drops to 0.2. Again resonance lockings which cause the square dips in the panel representing the tidal timescale (panel 6c) appear ubiquitous. The average increase of stellar rotation rates during the first $3 \mathrm{Myr}$ (see panel 6d) again causes the r-modes to become nearly resonant many times, and again the locking on to prograde modes responsible for the spin up at those instances is terminated by r-mode resonance crossings. Figure 7 indi- cates that also in this system locking takes place in at least one star most of the time, whereby new periods of locking start immediately after the previous locking is ended. Simultaneous locking in both stars appears frequently for the higher orbital eccentricity. The location of the stellar modes is shifted towards the higher orbital harmonics, which have become relatively stronger. The strip where resonance locking takes place still encompasses approximately the same g-modes, with a number of radial nodes between approximately 10 and 18 . Though the rate of orbital decay is lower in this case; the eccentricity is decreased by a quarter only, while in the case of an orbit with $P_{\text {orb }}=10$ days it was halved in only half the amount of time, we can still say that resonance locking is an important mechanism for orbital decay. It is only when no locking occurs that the timescale becomes much longer than the main sequence lifetime of the stars $(\approx 20 \mathrm{Myr})$, e.g. around time $t=4 \mathrm{Myr}$. 

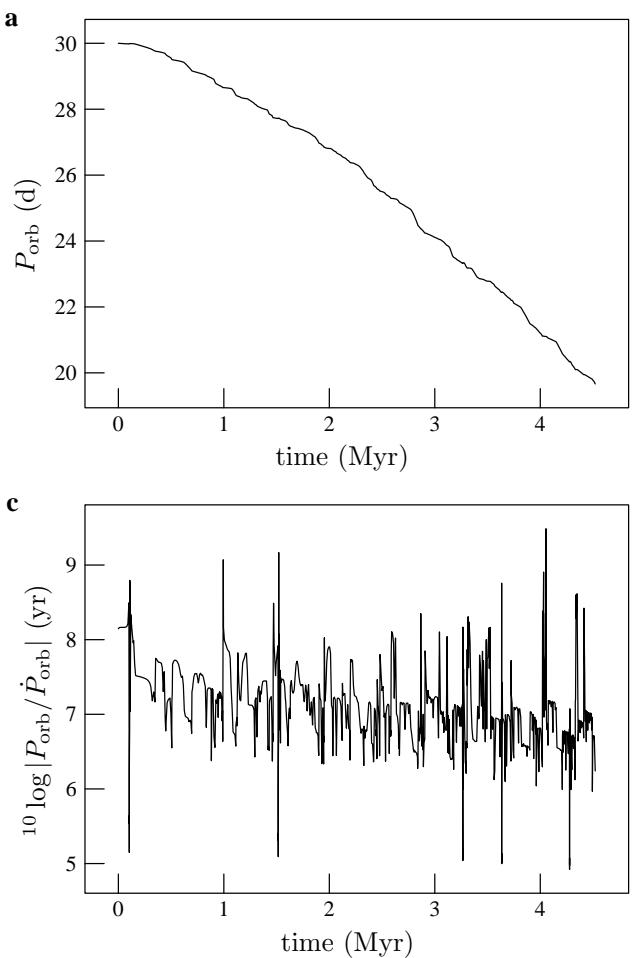
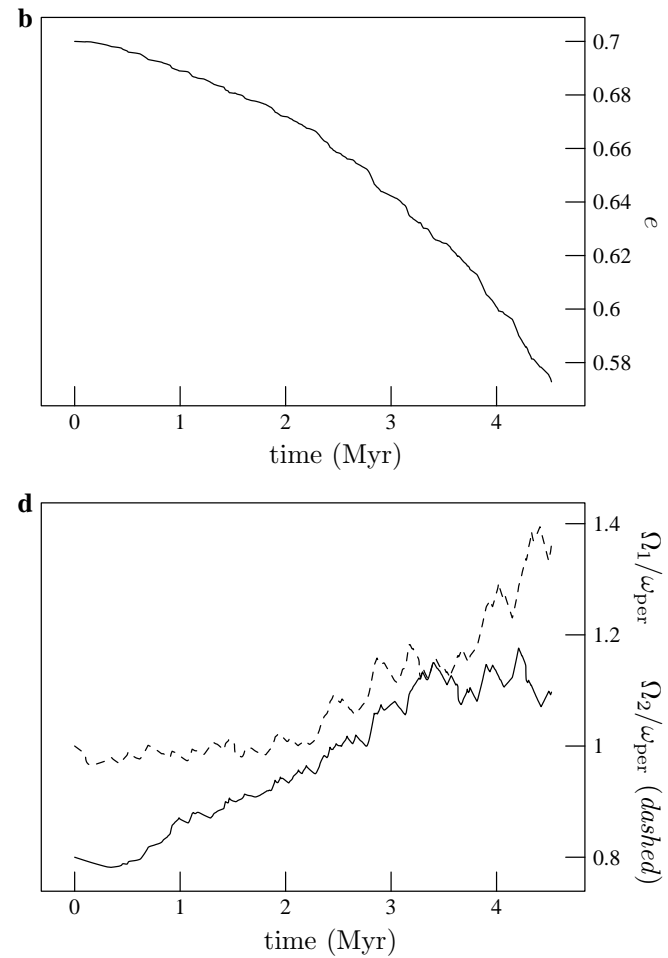

Fig. 8. At $t=0$ the orbit has a period $P_{\text {orb }}=30$ days (panel a)) and eccentricity $e=0.7$ (panel b)), and the stars are rotating at $80 \%$ and $100 \%$ of periastron frequency (panel $\mathbf{d}$ )). Periods of rapid orbital decay due to resonance locking are visible (square dips in panel c))

In this case the stellar rotation rates increase on average though the rate appears to level off near the end of the calculation. Though the rotation rates have not yet started decreasing, the stars have already acquired approximately equal rotation rates; this in contrast to the narrower orbit of Sect. 3.3.1, where the stars were found to rotate at equal rates only after these rates have started decreasing again.

From this example we see that the crucial issue in establishing conditions that are favourable for the occurrence of resonance locking lies in the balance between the strength of the orbital harmonics, which ultimately decreases with frequency, and the resonance peak height of the oscillation modes of the stars, which increases with frequency. Since in the current case of $P_{\text {orb }}=20$ days the mean orbital frequency $\omega$ is lower than that of the previous case in Sect. 3.3.1, those harmonics which have frequencies $n \omega$ near stellar g-modes that are sufficiently strong to establish locking now have higher $n$-values. To cause comparable resonance locking the Hansen coefficients $h_{n}$ for these higher orders should be similar to those of the lower order harmonics which caused locking in Sect. 3.3.1. A higher eccentricity is therefore necessary to establish comparable resonance locking in this wider system. Even though the established average timescale for the tidal decay is not as short as in the orbit with $P_{\text {orb }}=10$ days, resonance locking plays an important role in the evolution of this system. In the $59 \%$ of the evolution time during which locking takes place, $76 \%$ of the total eccentricity decline takes place.

\subsubsection{Orbit with $P_{\text {orb }}=30$ days}

Finally, an orbit with $P_{\text {orb }}=30$ days with even higher eccentricity $e=0.7$ is calculated (see Figs. 8 and 9 ). For such a configuration resonance locking appears more difficult to achieve; the duration of the locking periods is shorter, and the time in between phases of locking is longer. However, the tidal timescale during locking is again considerably shorter than outside locking, so even during these short periods significant tidal decay takes place. Locking occurs $39 \%$ of the time, accounting for $57 \%$ of the eccentricity decrease. Note that again the g-modes which are involved in the resonance locking are those that have approximately 15 radial nodes, and also that these g-mode frequencies now correspond to still higher harmonics of the orbit due to the decreased mean orbital frequency. Calculations of an orbit with $P_{\text {orb }}=30$ days and $e=0.65$ showed considerably less resonance locking, because for such lower eccentricity the high orbital harmonics are much weaker.

As the number of harmonics for which locking can occur remains large throughout the evolution, the small difference in evolution rates leads to quite different evolution for the two stars. Even after the rotation rates have become approximately equal around $t \simeq 3.5 \mathrm{Myr}$, different resonance locking in the two stars causes the stellar rotation rates to diverge near the end of the evolution.

Combined with the case of the orbit with $P_{\text {orb }}=5$ days presented in Sect. 3.2 these testcases show that resonance locking is a widespread phenomenon in the tidal evolution of eccentric binary systems, and that the mechanism 

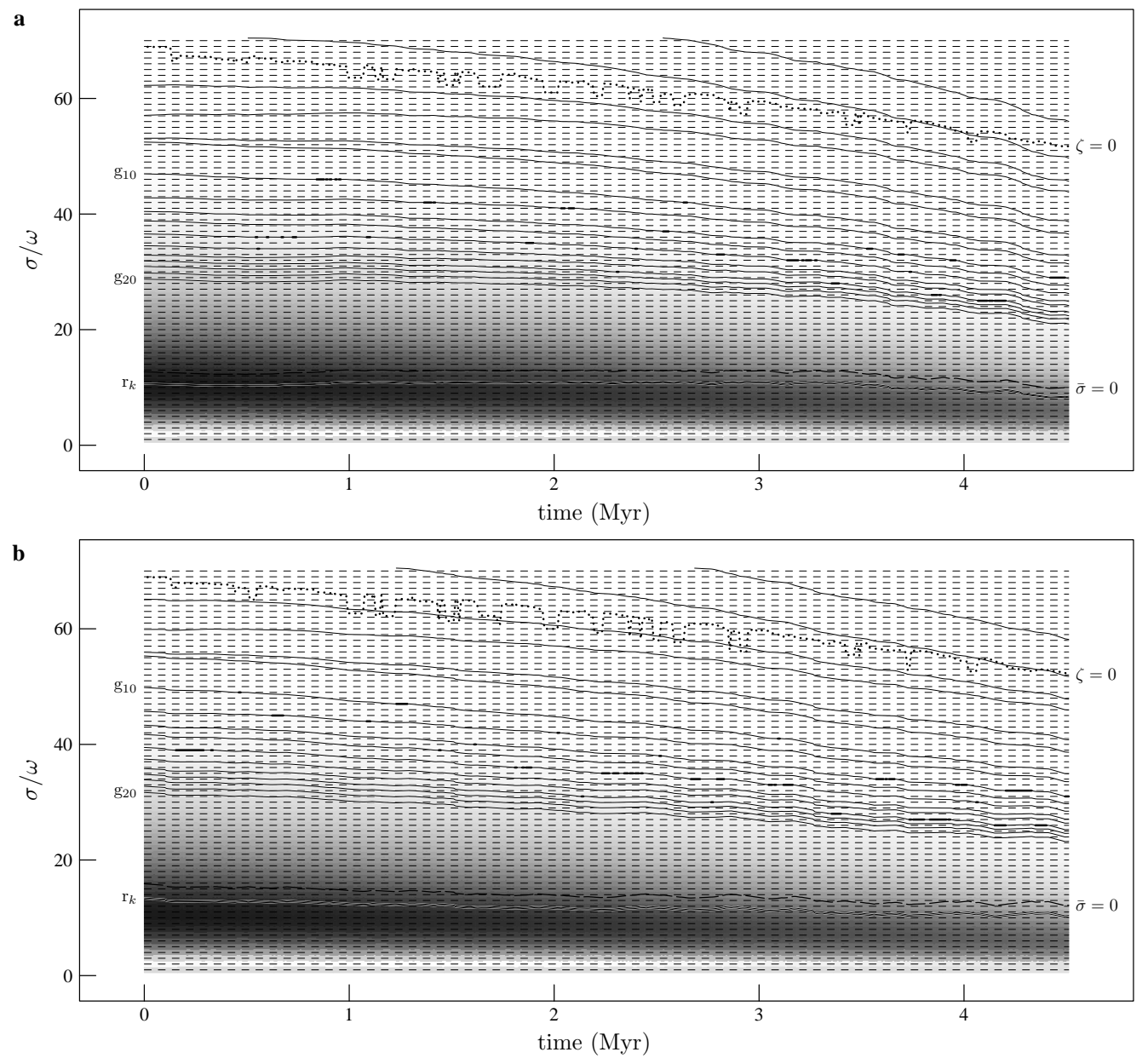

Fig. 9. For the orbital evolution pictured in Fig. 8 (initially $P_{\text {orb }}=30 \mathrm{~d}, e=0.7$ ), the solid curves show the stellar eigenfrequencies $\sigma_{0, i k}$ of $\operatorname{star} 1$ (panel $\left.\mathbf{a}\right)$ ) and star 2 (panel $\left.\mathbf{b}\right)$ ) normalised on the varying orbital frequency $\omega$. The normalised harmonic frequencies $\sigma_{n} / \omega=n$ of the orbit are plotted as horizontal dashed lines. The boundary between pro- and retrograde frequencies $(\bar{\sigma}=0)$ is given by the long dashes, and the relative strength of the different orbital harmonic components given by the Hansen coefficients $h_{n}$ is coded in greyscale. Thick lines mark resonance locking; the dotted line gives for the locked mode the normalised frequency for which $\zeta=0$. Resonance locking in at least one star is established during $39 \%$ of the time

significantly enhances orbital decay in orbits with periods up to thirty days. The details differ somewhat for the various orbital configurations; where the mildly eccentric orbit with period $P_{\text {orb }}=5$ days of Sect. 3.2, upon continued calculation, proved to quickly circularize in a few longlasting and intense stages of resonance locking, the wider and more eccentric orbits experience many more instances of resonance locking which last for relatively shorter time intervals.

\subsection{Uncertainties related to low frequency forcing}

For all orbits that have been calculated here the strongest orbital harmonics excite frequencies in the star that in the stellar frame are in between the lowest frequencies of the retro- and prograde g-modes of highest radial order $(k=20)$ that were taken into account. Except in those cases where the forcing occurs at frequencies very close to the r-modes, the torque on the star at these low fre- quencies is difficult to evaluate numerically, because of the short wavelength of the response and the consequent poor spatial resolution. To estimate how these uncertainties translate into uncertainties in the conclusions of this work, we recalculate the orbital evolution of the orbit with $P_{\text {orb }}=10$ days shown in Fig. 4, whereby the constant torque level that was assumed to describe the response at these low frequencies is diminished by an order of magnitude, i.e. we now adopt $\mathcal{T}_{\text {low }}=310^{36} \mathrm{cgs}$. The resulting evolution is shown in Figs. 10 and 11. As can be seen in these plots, the resonance locking mechanism does depend on the strength of the low frequency stellar response. This is because the low order retrograde harmonics give rise to stellar spin down which can drive the prograde modes into resonance. This can be seen in Fig. 3 where the dash-dotted lines give the contribution of the combined orbital harmonic frequencies that do not experience locking, which for the largest part consists of contributions by these low order retrograde harmonics. Decreasing the 
a
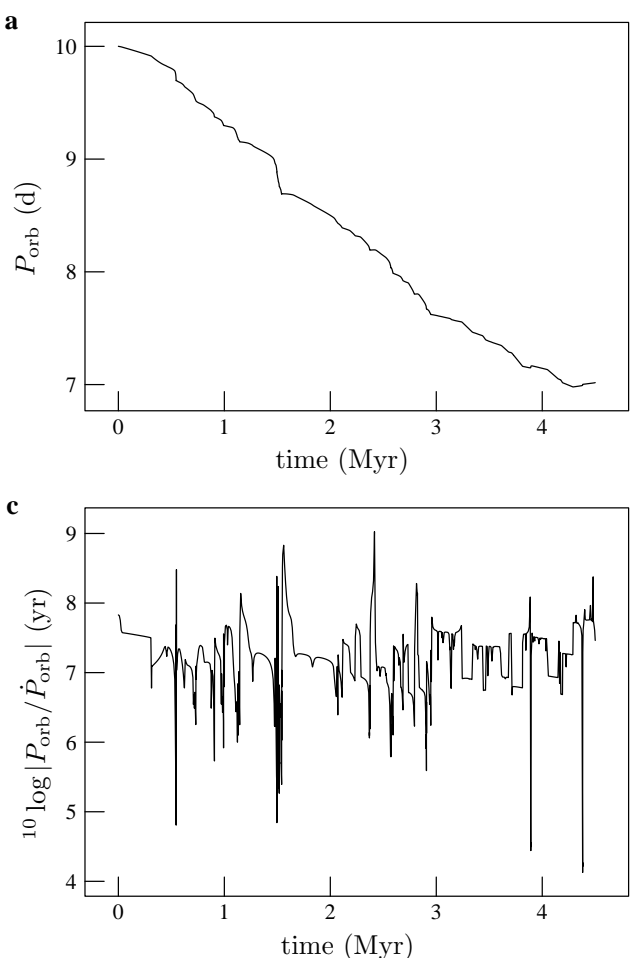
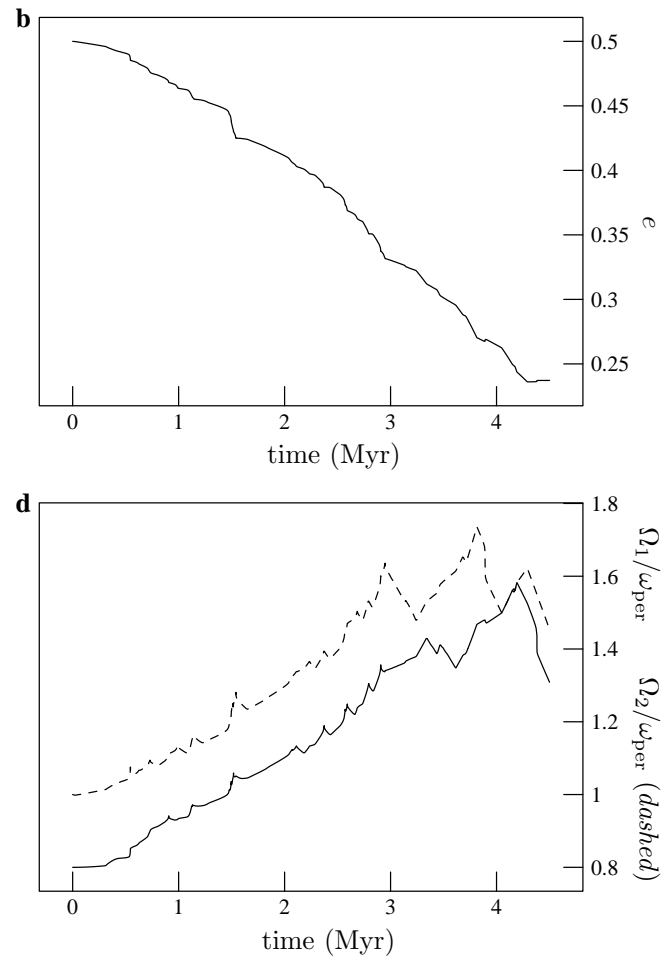

Fig. 10. At $t=0$ the orbit has a period $P_{\text {orb }}=10$ days (panel a)) and eccentricity $e=0.5$ (panel b)). The stars rotate initially at $80 \%$ and $100 \%$ of periastron frequency (panel d)). Resonance locking causes many periods of rapid orbital decay (square dips in panel c)), during which the locked star(s) spins up. Compared to the case shown in Fig. 4 the torque level at low frequencies is reduced by a factor $10: \mathcal{T}_{\text {low }}=310^{36}(\mathrm{cgs})$. The time interval needed to establish comparable changes in the orbit is now more than twice as long

torque level for these low frequencies implies that the balance for resonance locking demands a weaker excitation of the locked prograde mode, with a correspondingly longer evolution timescale. Also, the rate at which harmonics drift through the stellar spectrum is lower, so the chance of quickly running into the next locking is smaller. Indeed, compared to the standard case described in Sect. 3.3.1, the evolution shows longer periods where no locking occurs, and the locking that does occur results in longer tidal timescales. In this case of reduced low frequency torques locking occurs $58 \%$ of the time, causing $71 \%$ of the total eccentricity change.

Decreasing the low frequency stellar response even further to a negligible level, such that the low order orbital harmonics can effectively be disregarded, resonance locking becomes yet somewhat weaker, but not by a significant fraction. The reason is that the continuous stellar spin down due to stellar evolution (see Eq. (19)) becomes important at these levels, and that contribution is independent of the low frequency stellar response. Consequently, we can conclude that resonance locking occurs frequently during the orbital evolution of eccentric binaries, but that its actual effectiveness depends on the somewhat uncertain tidal torque levels for low forcing frequencies in rotating stars.

\subsection{Non-linear effects}

The duration and maximum intensity of each resonance locking phase depends critically on the height of the resonance peak involved; the linear analysis we used to calculate the stellar response may not be sufficiently accurate if resonance locking occurs for large amplitude oscillations. However, tuned by the balance in Eq. (20) a locked harmonic $n$ will during resonance locking stay at a relatively low peak height $\mathcal{Q}_{n}$ as long as its corresponding Hansen coefficient $h_{n}$ remains adequate. Resonance locking thus tends to occur with rather limited oscillation amplitudes. In a system with $P_{\text {orb }}$ between 5 and 10 days the steady state amplitude of the dimensionless density perturbation $\rho^{\prime} / \rho$ in the stellar interior during locking is typically a fraction of a percent (for the standard low frequency torque level $\left.\mathcal{T}_{\text {low }}=310^{37} \mathrm{cgs}\right)$. Nonlinear effects are therefore not likely to be very significant for the locked mode.

The amplitude of the surface displacement vector $\boldsymbol{\xi}$ in units of the stellar radius $R_{i}$ is of the same order as the dimensionless density perturbation, which translates into a surface oscillation velocity amplitude of less than $1 \mathrm{~km} \mathrm{~s}^{-1}$ during locking. Observation of a locked mode may therefore require high data quality.

Nonlinear effects will play a role during resonance crossings of strong low order orbital harmonics which pull themselves through resonance (e.g. r-modes), and for 

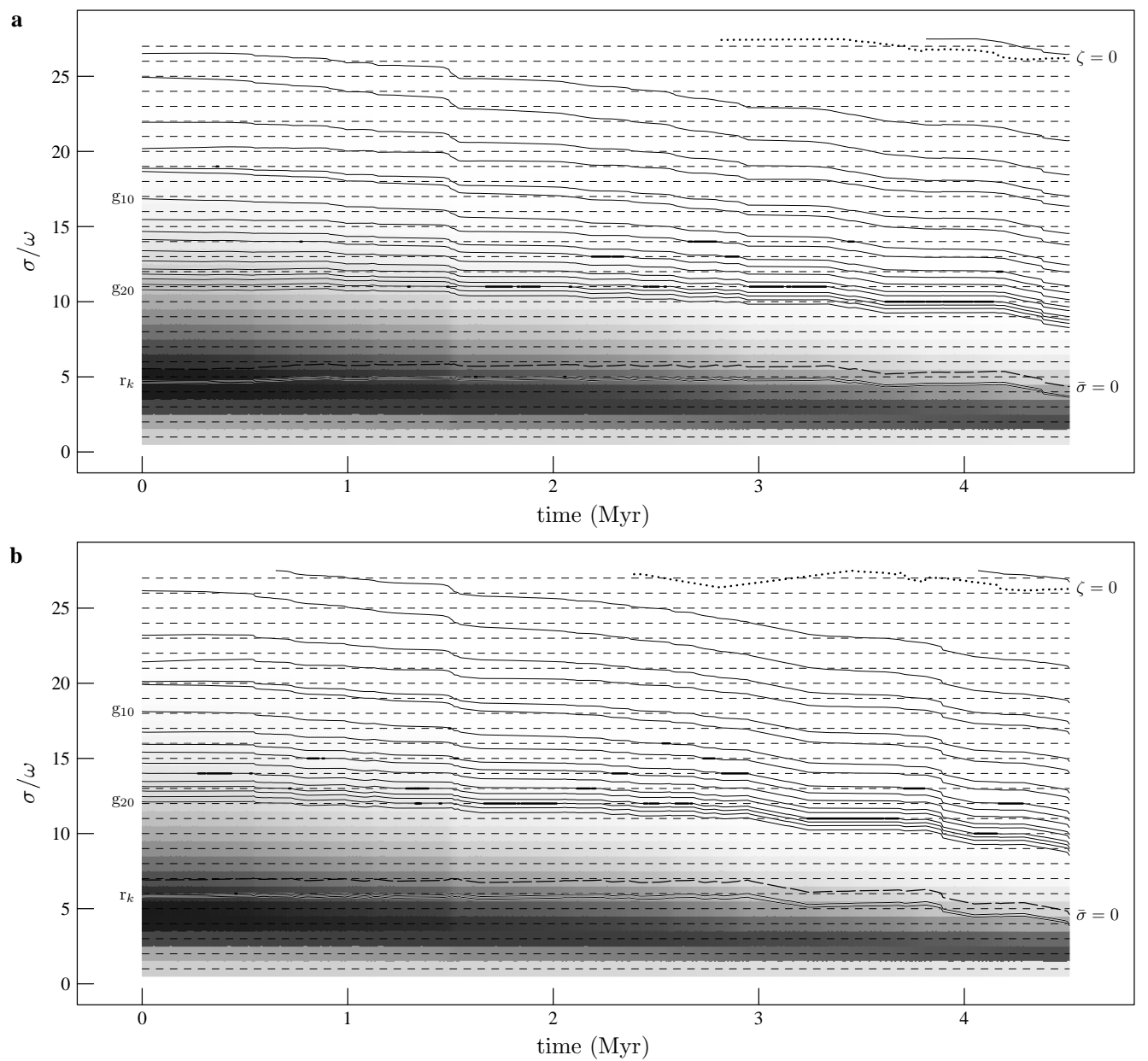

Fig. 11. For the orbital evolution pictured in Fig. 10 (initially $P_{\text {orb }}=10 \mathrm{~d}, e=0.5$, $\mathcal{T}_{\text {low }}=310^{36}$ (cgs)), the solid curves show the stellar eigenfrequencies $\sigma_{0, i k}$ of star 1 (panel a)) and star 2 (panel b)) normalised on the varying orbital frequency $\omega$. The normalised harmonic frequencies $\sigma_{n} / \omega=n$ of the orbit are plotted as horizontal dashed lines. The boundary between pro- and retrograde frequencies $(\bar{\sigma}=0)$ is given by the long dashes, and the relative strength of the different orbital harmonic components given by the Hansen coefficients $h_{n}$ is coded in greyscale. Thick lines mark resonance locking; the dotted line gives for the locked mode the normalised frequency for which $\zeta=0$. Resonance locking in at least one star is established during $58 \%$ of the time

which no resonance locking can occur. The duration of such resonance crossings is, however, short so that the tidal exchange of angular momentum and energy is limited. During these fast resonance crossings the steady state approximation may break down. The oscillation amplitudes during these fast crossings can become large, so these crossings could possibly induce large scale mixing. This mixing would change the structure of the star and enlarge the reservoir of nuclear fuel so the MS lifetime of the stars could be extended significantly. As the oscillation characteristics of a star depend on its structure, the effect of mixing feeds back into the details of the tidal evolution. Another interesting property of nonlinear oscillations is that the eigenfrequency of an excited mode depends on its amplitude, so while becoming resonant with an orbital harmonic, the stellar mode would intrinsically start shifting, not only as a result of changes in the orbit or in the stellar rotation rate. This would make the locking mecha- nism certainly even more interesting (and complex), and could possibly change the resonance crossing mechanism of the strong low order harmonics for which the amplitudes may be large. Inclusion of nonlinear terms should be considered for future work, but lies beyond the scope of the work currently presented.

\section{Conclusion}

By performing calculations of the orbital evolution of binary systems consisting of two early type MS stars with eccentric orbits we have demonstrated the importance of weak high frequency harmonic components of the perturbing tidal potential, which resonantly excite stellar g-mode oscillations. The resonance locking mechanism which was investigated in a previous paper for an early type MS star with a compact companion (Paper II) is shown to be enhanced by the fact that in a double MS binary dissipation 
in both stars influences the orbit. It is shown that for a large range of orbital periods and eccentricities resonance locking is such a common phenomenon that at any given time the chance that at least one star will be involved in a stage of near resonant tidal excitation is significant. This has large implications for the decay rate of the eccentricity and orbital period, which becomes much enhanced. For early type MS binaries with orbital periods less than about 10 days the orbital eccentricity is predicted to decay from 0.6 to 0.2 in only a few million years.

Acknowledgements. This work was sponsored by the Stichting Nationale Computerfaciliteiten (National Computing Facilities Foundation, NCF) for the use of supercomputing facilities, with financial support from the Netherlands Organisation for Scientific Research (NWO) and by NWO Spinoza grant 08-0 to E. P. J. van den Heuvel.

\section{References}

De Cat, P., Aerts, C., De Ridder, J., et al 2000, A\&A, 355, 1015

Giuricin, G., Mardirossian, F., \& Mezzetti, M. 1984, A\&A, 134, 365

Harmanec, P., Hadrava, P., Yang, S., et al 1997, A\&A, 319, 867

Papaloizou, J. C. B., \& Savonije, G. J. 1997, MNRAS, 291, 651

Savonije, G. J., \& Papaloizou, J. C. B. 1983, MNRAS, 203, 581

Savonije, G. J., \& Papaloizou, J. C. B. 1997, MNRAS, 291, 633

Smith, M. A. 1985a, ApJ, 297, 206

1985b, ApJ, 297, 224

Willems, B., Van Hoolst, T., Smeyers, P., \& Waelkens, C. 1997, A\&A, 326, L37

Witte, M. G., \& Savonije, G. J. 1999a, A\&A, 341, 842

Witte, M. G., \& Savonije, G. J. 1999b, A\&A, 350, 129

Zahn, J. P. 1977, A\&A, 57, 383 